\title{
Chromosome contacts in activated T cells identify autoimmune disease candidate genes
}

\author{
Oliver S. Burren 1,2, Arcadio Rubio García ${ }^{2,3}$, Biola-Maria Javierre ${ }^{4}$, Daniel B. Rainbow ${ }^{2,3}$, Jonathan Cairns ${ }^{4}$, \\ Nicholas J. Cooper², John J. Lambourne ${ }^{5}$, Ellen Schofield ${ }^{2}$, Xaquin Castro Dopico ${ }^{2}$, Ricardo C. Ferreira, ${ }^{2,3}$, \\ Richard Coulson ${ }^{2}$, Frances Burden ${ }^{5,6}$, Sophia P. Rowlston ${ }^{5,6}$, Kate Downes ${ }^{5,6}$, Steven W. Wingett ${ }^{4}$, Mattia Frontini ${ }^{5,6,7}$, \\ Willem H. Ouwehand ${ }^{5,6,7,8}$, Peter Fraser ${ }^{4}$, Mikhail Spivakov ${ }^{4}$, John A. Todd ${ }^{2,3}$, Linda S. Wicker ${ }^{2,3}$, \\ Antony J. Cutler 2,3 and Chris Wallace 1,2,9* $^{*}$
}

\begin{abstract}
Background: Autoimmune disease-associated variants are preferentially found in regulatory regions in immune cells, particularly $\mathrm{CD}^{+} \mathrm{T}$ cells. Linking such regulatory regions to gene promoters in disease-relevant cell contexts facilitates identification of candidate disease genes.

Results: Within $4 \mathrm{~h}$, activation of $\mathrm{CD}^{+}{ }^{+} \mathrm{T}$ cells invokes changes in histone modifications and enhancer RNA transcription that correspond to altered expression of the interacting genes identified by promoter capture Hi-C. By integrating promoter capture $\mathrm{Hi}-\mathrm{C}$ data with genetic associations for five autoimmune diseases, we prioritised 245 candidate genes with a median distance from peak signal to prioritised gene of $153 \mathrm{~kb}$. Just under half (108/245) prioritised genes related to activation-sensitive interactions. This included IL2RA, where allele-specific expression analyses were consistent with its interaction-mediated regulation, illustrating the utility of the approach.
\end{abstract}

Conclusions: Our systematic experimental framework offers an alternative approach to candidate causal gene identification for variants with cell state-specific functional effects, with achievable sample sizes.

Keywords: Genetics, Genomics, Chromatin conformation, $\mathrm{CD}^{+} \mathrm{T}$ cells, $\mathrm{CD} 4^{+} \mathrm{T}$ cell activation, Autoimmune disease, Genome-wide association studies

\section{Background}

Genome-wide association studies (GWAS) in the last decade have associated 324 distinct genomic regions to at least one and often several autoimmune diseases (https:// www.immunobase.org). The majority of associated variants lie outside genes [1] and presumably tag regulatory variants

\footnotetext{
* Correspondence: cew54@cam.ac.uk

Oliver S. Burren, Arcadio Rubio García, Biola-Maria Javierre, and Daniel B. Rainbow contributed equally to this work.

John A. Todd, Linda S. Wicker, Antony J. Cutler, and Chris Wallace contributed equally to this work.

'Department of Medicine, University of Cambridge, Addenbrooke's Hospital, Cambridge CB2 OSP, UK

${ }^{2}$ JDRF/Wellcome Trust Diabetes and Inflammation Laboratory, Department of Medical Genetics, NIHR Cambridge Biomedical Research Centre, Cambridge Institute for Medical Research, University of Cambridge, Cambridge CB2 OXY, UK

Full list of author information is available at the end of the article
}

acting on nearby or more distant genes [2, 3]. Progress from GWAS discovery to biological interpretation has been hampered by lack of systematic methods to define the gene(s) regulated by a given variant. The use of $\mathrm{Hi}-\mathrm{C}$ [4] and capture Hi-C to link GWAS identified variants to their target genes for breast cancer [5] and autoimmune diseases [6] using cell lines, has highlighted the potential for mapping long-range interactions in advancing our understanding of disease association. The observed cell specificity of these interactions indicates a need to study primary disease-relevant human cells and investigate the extent to which cell state may affect inference.

Integration of GWAS signals with cell-specific chromatin marks has highlighted the role of regulatory variation in immune cells [7], and in particular $\mathrm{CD}_{4}^{+} \mathrm{T}$ cells, in autoimmune diseases [8]. Concordantly, differences in 
DNA methylation of immune-related genes have been observed in $\mathrm{CD}^{+} \mathrm{T}$ cells from autoimmune disease patients compared to healthy controls $[9,10] . \mathrm{CD}^{+} \mathrm{T}$ cells are at the centre of the adaptive immune system and exquisite control of activation is required to guide $\mathrm{CD} 4^{+} \mathrm{T}$ cell fate through selection, expansion and differentiation into one of a number of specialised subsets. Additionally, the prominence of variants in physical proximity to genes associated with $\mathrm{T}$ cell regulation in autoimmune disease GWAS and the association of human leukocyte antigen haplotypes have suggested that control of $\mathrm{T}$ cell activation is a key etiological pathway in development of many autoimmune diseases [11].

We explored the effect of activation on $\mathrm{CD}^{+} \mathrm{T}$ cell gene expression, chromatin states and chromosome conformation. Promoter capture $\mathrm{Hi}-\mathrm{C}(\mathrm{PCHi}-\mathrm{C})$ was used to map promoter interacting regions (PIRs) and to relate activation-induced changes in gene expression to changes in chromosome conformation and transcription of (PCHi-C) linked enhancer RNAs (eRNAs). We also finemapped the most probable causal variants for five autoimmune diseases, autoimmune thyroid disease (ATD), coeliac disease (CEL), rheumatoid arthritis (RA), systemic lupus erythematosus (SLE) and type 1 diabetes (T1D). We integrated these sources of information to derive a systematic prioritisation of candidate autoimmune disease genes.

\section{Results}

\section{A time-course expression profile of early $\mathrm{CD} 4^{+} \mathrm{T}$ cell activation}

We profiled gene expression in $\mathrm{CD}^{+} \mathrm{T}$ cells from 20 healthy individuals across a 21 hour (h) activation timecourse and identified eight distinct gene modules by clustering these profiles (Fig. 1, Additional file 1: Table S1). This experimental approach focused on much earlier events than previous large time-course studies (e.g. $6 \mathrm{~h}-$ 8 days [12]) and highlights the earliest changes that are either not seen after or are returning towards baseline by $6 \mathrm{~h}$ (Additional file 2: Figure S1). Gene set enrichment analysis using MSigDB Hallmark gene sets [13] demonstrated that these modules captured temporally distinct aspects of $\mathrm{CD} 4^{+} \mathrm{T}$ cell activation. For example, negative regulators of TGF-beta signalling were rapidly upregulated and returned to baseline by $4 \mathrm{~h}$. Interferon responses, inflammatory responses and IL-2 and STAT5 signalling pathways showed a more sustained upregulation beyond $6 \mathrm{~h}$, while fatty acid metabolism was initially downregulated in favour of oxidative phosphorylation.

PCHi-C captures dynamic enhancer-promoter interactions We examined activated and non-activated $\mathrm{CD}^{+}{ }^{+} \mathrm{T}$ cells purified from healthy individuals in more detail at the 4-h time point, at which the average fold change of genes related to cytokine signalling and inflammatory response was maximal, using total RNA sequencing (RNA-seq), histone mark chromatin immunoprecipitation sequencing (ChIP-seq), and PCHi-C. Of 8856 genes identified as expressed (see 'Methods') in either condition (non-activated or activated), $25 \%$ were upregulated or downregulated (1235 and 952 genes, respectively, false discovery rate $(\mathrm{FDR})<0.01$, Additional file 3: Table S2). We used $\mathrm{PCHi}-\mathrm{C}$ to characterise promoter interactions in activated and non-activated $\mathrm{CD} 4^{+} \mathrm{T}$ cells. Our capture design baited 22,076 HindIII fragments (median length, $4 \mathrm{~kb}$ ) which contained the promoters of 29,131 annotated genes, 18,202 of which are protein-coding (Additional file 4: Table S3). We detected 283,657 unique PCHi-C interactions with the CHiCAGO pipeline [14], with $55 \%$ found in both activation states and $22 \%$ and $23 \%$ found only in non-activated and only in activated $\mathrm{CD}_{4}^{+} \mathrm{T}$ cells, respectively (Additional file 5: Table S4). Of the baited promoter fragments, 11,817 were involved in at least one interaction, with a median distance between interacting fragments of $324 \mathrm{~kb}$. Each interacting promoter fragment connected to a median of eight PIRs, while each interacting PIR was connected to a median of one promoter fragment (Additional file 2: Figure S2).

We compared our interaction calls to an earlier ChIAPET dataset from non-activated $\mathrm{CD}^{+}{ }^{+} \mathrm{T}$ cells [15] and found we replicated over $50 \%$ of the longer-range interactions (100 kb or greater), with replication rates over tenfold greater for interactions found in non-activated $\mathrm{CD}^{+} \mathrm{T}$ cells compared to interactions found only in erythroblasts or megakaryocytes (Additional file 2: Figure S3). We also compared histone modification profiles in interacting fragments in $\mathrm{CD}_{4}^{+} \mathrm{T}$ cells to interacting fragments found in erythroblasts or megakaryocytes. Both promoter fragments and, to a lesser extent, PIRs were enriched for histone modifications associated with transcriptionally active genes and regulatory elements (H3K27ac, H3K4me1, H3K4me3; Additional file 2: Figure S4) and changes in H3K27ac modifications at both promoter fragments and PIRs correlated with changes in gene expression upon activation. PIRs, but not promoter fragments, showed overlap with regions previously annotated as enhancers [16].

We found that absolute levels of gene expression correlated with the number of PIRs (Additional file 2: Figure S5a; rho, 0.81), consistent with recent observations $[15,17]$. We defined a subset of PCHi-C interactions that were specifically gained or lost upon activation (2334 and 1866, respectively, FDR < 0.01) and found that the direction of change (gain or loss) at these differential interactions agreed with the direction of differential expression (upregulated or downregulated) at the module level (Fig. 2). We further found that dynamic changes in gene expression upon activation correlated with changing numbers of PIRs. Notably, the pattern was 

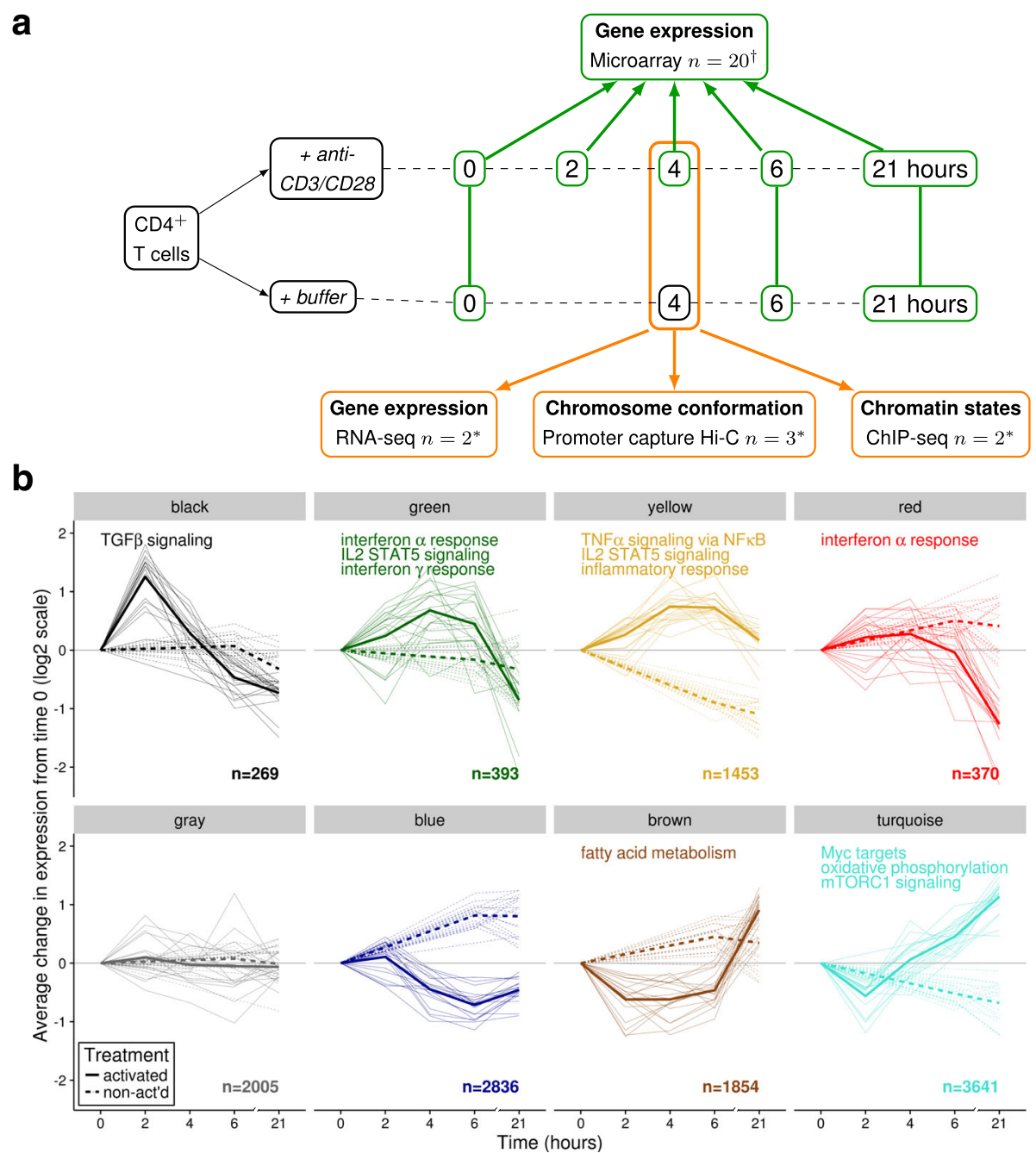

Fig. 1 a Summary of genomic profiling of $C D 4^{+} T$ cells during activation with anti-CD3/CD28 beads. We examined gene expression using microarray in activated and non-activated CD4 ${ }^{+} T$ cells across $21 \mathrm{~h}$ and assayed cells in more detail at the 4-h time point using ChIP-seq, RNA-seq and PCHi-C. $\mathrm{n}$ gives the number of individualst or pools* assayed. $\mathbf{b}$ Eight modules of co-regulated genes were identified and eigengenes are plotted for each individual (solid lines=activated, dashed lines= non-activated), with heavy lines showing the average eigengene across individuals. We characterised these modules by gene set enrichment analysis within the MSigDB HALLMARK gene sets; where significant gene sets were found, up to three shown per module. $n$ is the number of genes in each module

asymmetrical, with a gained interaction associating with approximately twofold the change associated with a lost interaction (Fig. 3a). Given the $>6$-h median half-life of messenger RNAs (mRNAs) expressed in T cells [18] (Additional file 2: Figure S5b), it is possible that the relatively smaller changes associated with lost interactions are due to the persistence of downregulated transcripts at the early stages of $\mathrm{T}$ cell activation.

As we sequenced total RNA without a poly(A) selection step, we were able to detect regulatory region RNAs (regRNAs), which are generally non-polyadenylated and serve as a mark for promoter and enhancer activity [19]. We defined 6147 'expressed' regRNAs (see 'Methods') that mapped within regulatory regions defined by a 15 state
ChromHMM [20] model (Additional file 2: Figure S6) and validated them by comparison to an existing cap analysis of gene expression (CAGE) dataset [21] which has been successfully used to catalogue active enhancers [22]. We found 2888/3897 (74\%) regRNAs expressed in nonactivated cells overlap CAGE defined enhancers. This suggests that the combination of chromatin state annotation and total RNA-seq data presents an alternative approach to capture active enhancers.

Almost half (48\%) of expressed regRNAs showed differential expression after activation (2254/681 upregulated/downregulated; FDR $<0.01$ ). To determine whether activity at these regRNAs could be related to that at PCHi-C linked genes, we focused attention on a subset 

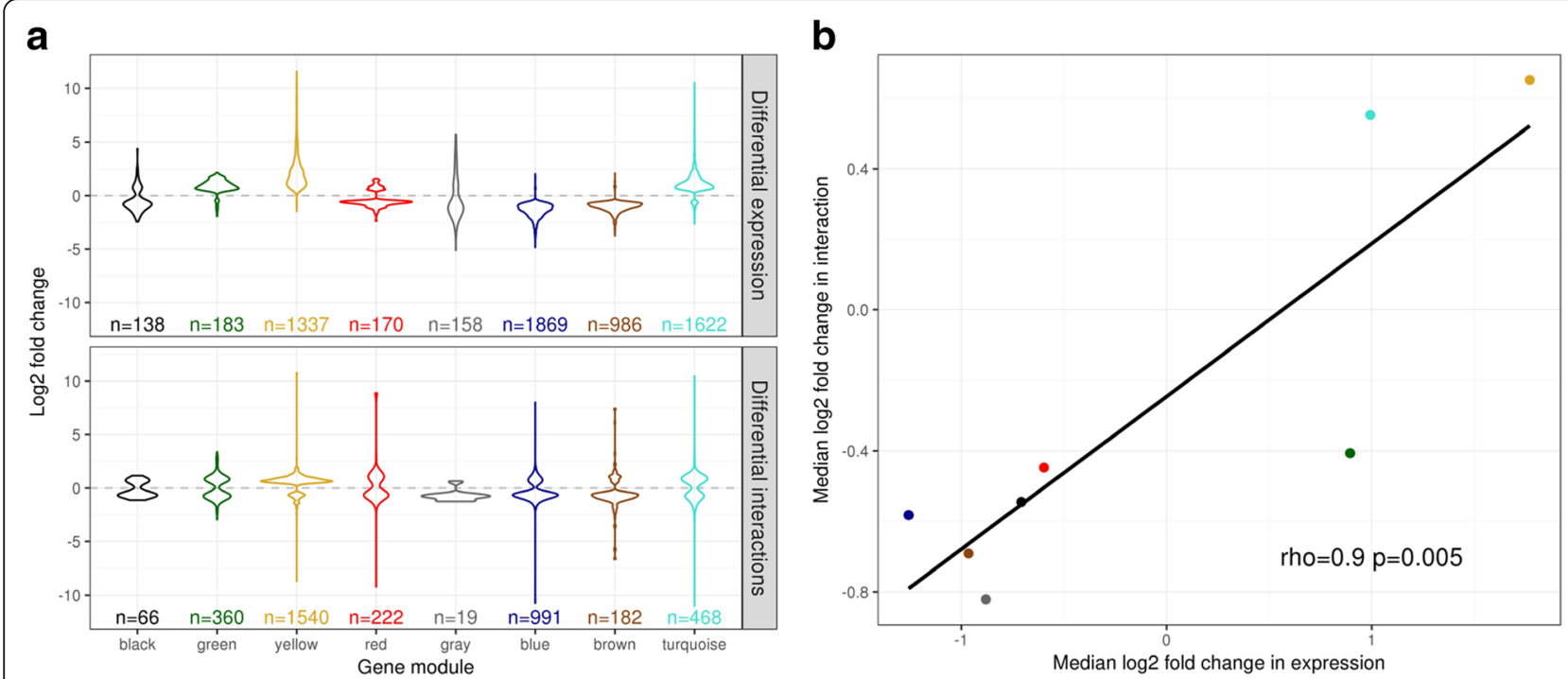

Fig. 2 Change in PCHi-C interactions correlate with change in gene expression. a Distribution of significant (FDR $<0.01)$ fold changes induced by activation of $\mathrm{CD}^{+} \mathrm{T}$ cells in (top) gene expression and (bottom) differential PCHi-C interactions for differentially expressed genes in by module. b Median significant expression and interaction fold changes by module are correlated (Spearman rank correlation)

of 640 intergenic regRNAs, which correspond to a definition of eRNAs [23]. Of these, 404 (63\%) overlapped PIRs detected in $\mathrm{CD}^{+} \mathrm{T}$ cells and we found significant agreement in the direction of fold changes at eRNAs and their $\mathrm{PCHi}-\mathrm{C}$ linked protein-coding genes in activated $\mathrm{CD} 4^{+} \mathrm{T}$ cells $(p<0.0001$, Fig. 3b). We also observed a synergy between regRNA expression and the estimated effect of a PIR on expression with a gain or loss of a PIR overlapping a differentially regulated regRNA having the largest estimated effect on gene expression (Fig. 3c), supporting a sequential model of gene activation [24]. While regRNA function is still unknown [23], our results demonstrate the detection, by $\mathrm{PCHi}-\mathrm{C}$, of condition-specific connectivity between promoters and enhancers involved coordinating gene regulation.

\section{PCHi-C-facilitated mapping of candidate disease-causal genes}

We defined an experimental framework to integrate PCHi-C interactions with GWAS data to map candidate disease-causal genes for autoimmune diseases (Fig. 4). First, to confirm that $\mathrm{PCHi}-\mathrm{C}$ interactions inform interpretation of autoimmune disease GWAS, we tested whether PIRs were enriched for autoimmune disease GWAS signals by testing for different distributions of GWAS $p$ values in PIRs of activated or non-activated $\mathrm{CD} 4^{+} \mathrm{T}$ cells compared to non-lymphoid cells (megakaryocytes and erythroblasts) and then in PIRs of activated compared to non-activated $\mathrm{CD} 4^{+} \mathrm{T}$ cells. To perform the test, we used blockshifter [17] which accounts for correlation between (1) neighbouring variants in the GWAS data and (2) neighbouring HindIII fragments in the interacting data by rotating one dataset with respect to the other, as previously proposed [25]. This method appropriately controls type 1 error rates, in contrast to methods based on counting associated SNP/PIRs which ignore correlation, such as a Fisher's exact test (Additional file 2: Figure S7). We found autoimmune GWAS signals were enriched in $\mathrm{CD} 4^{+} \mathrm{T}$ cell PIRs compared to non-autoimmune GWAS signals (Wilcoxon $p=$ $2.5 \times 10^{-7}$ ) and preferentially so in activated vs. nonactivated cells (Wilcoxon $p=4.8 \times 10^{-5}$; Fig. 4).

Next, we fine-mapped causal variants for five autoimmune diseases using genetic data from a dense targeted genotype array, the ImmunoChip (ATD, CEL, RA, T1D) and summary data from GWAS data imputed to $1000 \mathrm{Ge}$ nomes Project (RA, SLE; Additional file 6: Table S5). For the ImmunoChip datasets, with full genotype data, we also imputed to 1000 Genomes and used a Bayesian finemapping approach [26], which avoids the need for stepwise regression or assumptions of single causal variants and which provides a measure of posterior belief that any given variant is causal by aggregating posterior support over models containing that variant. Variant-level results are given in Additional files 7 and 8: Tables S6a and S6b, and show that of 73 regions with genetic association signals to at least one disease (minimum $p<5 \times 10^{-8}$; 106 disease associations), nine regions have strong evidence that they contain more than one causal variant (posterior probability $>0.5$ ), among them the well-studied region on chromosome 10 containing the candidate gene IL2RA [26]. For the GWAS summary data, we make the simplifying assumption that there exists a single causal variant in 


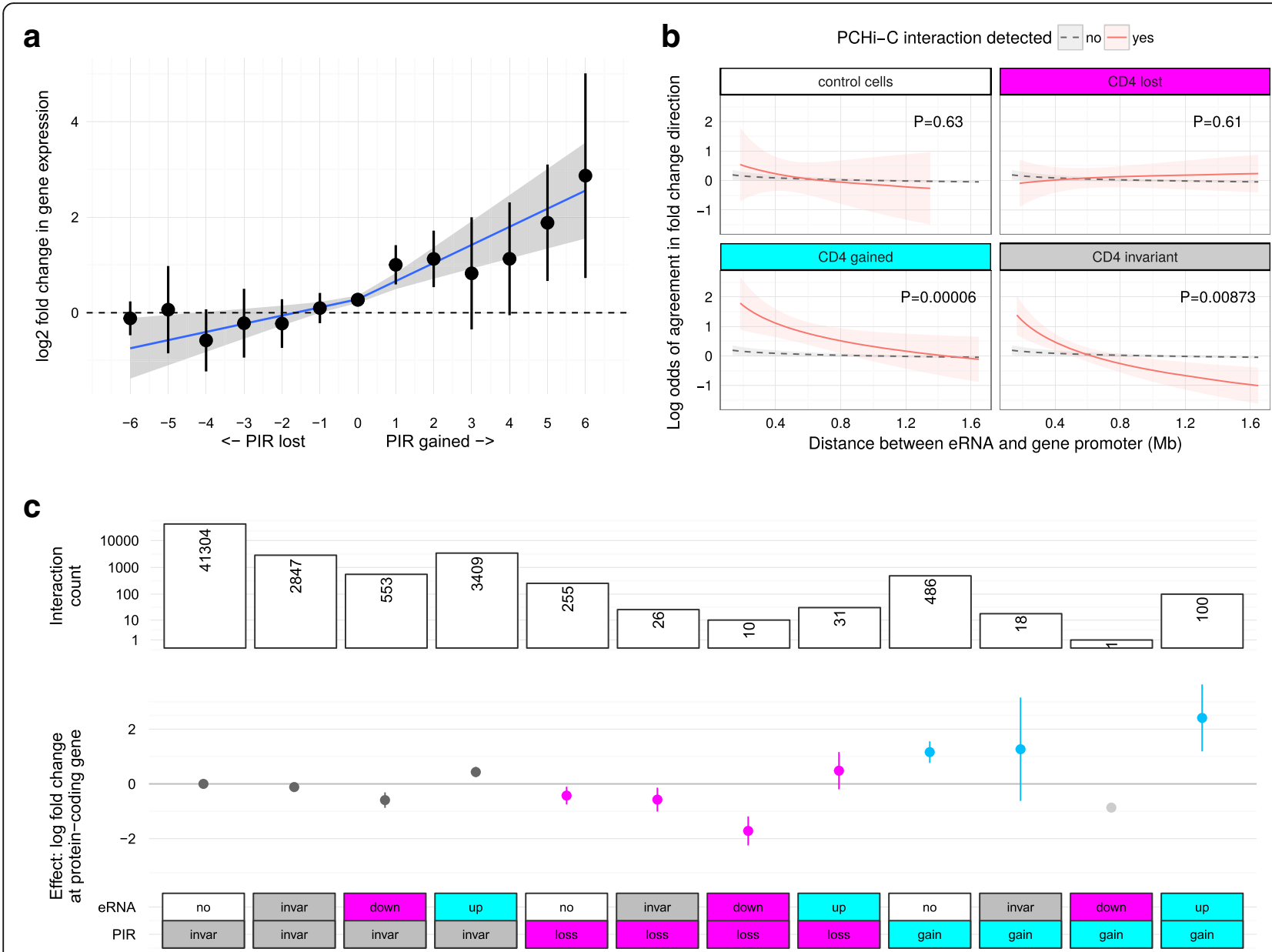

Fig. $3 \mathrm{PCHi}-\mathrm{C}$ interactions and enhancer activity predict change in gene expression. a Change in gene expression at protein coding genes ( $\log _{2}$ fold change, $y$-axis) correlates with the number of PIRs gained or lost upon activation (x-axis). b Fold change at transcribed sequence within the intergenic subset of regulatory regions ('eRNAs') was more likely to agree with the direction of protein-coding gene fold change when the two are linked by PCHi-C (red) in activated $\mathrm{CD} 4^{+} \mathrm{T}$ cells compared to pairs of eRNA and protein-coding genes formed without regard to PCHi-C derived interactions (background, grey, $p<10^{-4}$ ). Interactions were categorised as control (present only in megakaryocytes and erythroblasts, our control cells), invariant ('invar'; present in non-activated and activated CD4 ${ }^{+} \mathrm{T}$ cells), 'loss' (present in non-activated but not activated CD4 ${ }^{+} \mathrm{T}$ cells and significantly downregulated at FDR $<0.01$ ) or 'gain' (present in activated but not non-activated $\mathrm{CD}^{+} \mathrm{T}$ cells and significantly upregulated at FDR < 0.01). c Gain or loss of PIRs upon activation predicts change in gene expression, with the estimated effect more pronounced if accompanied by upregulation or downregulation at an eRNA. Points show estimated effect on gene expression of each interaction and lines the $95 \%$ confidence interval. PIRs categorised as in (b). eRNAs categorised as no (undetected), invariant ('invar', detected in non-activated and activated CD4 ${ }^{+} \mathrm{T}$ cells, differential expression FDR $\geq 0.01$ ), up (upregulated; FDR $<0.01$ ) or down (downregulated; FDR $<0.01$ ). Bar plot (top) shows the number of interactions underlying each estimate. Note that eRNA = down, PIR = gain (light gray) has only one observation so no confidence interval can be formed and is shown for completeness only

any LD-defined genetic region and again generate posterior probabilities that each variant is causal [27]. To integrate these variant level data with $\mathrm{PCHi}-\mathrm{C}$ interactions and prioritise protein-coding genes as candidate causal genes for each autoimmune disease, for each gene, we aggregated posterior support over all models containing variants in PIRs for the gene promoter, within the promoter fragment or within its immediate neighbour fragments. Neighbouring fragments are included because of limitations in the ability of $\mathrm{PCHi}-\mathrm{C}$ to detect very proximal interactions (within a region consisting of the promoter baited fragment and one Hin$d$ III fragment either side). The majority of gene scores were close to $0(99 \%$ of genes have a score $<0.05)$ and we chose to use a threshold of 0.5 to call genes prioritised for further investigation. Having both ImmunoChip and summary GWAS data for RA allowed us to compare the two methods. Overlap was encouraging: of 24 genes prioritised for RA from ImmunoChip, 20 had a gene score > 0.5 in the GWAS prioritisation, and a further three had gene scores $>0.2$. The remaining gene, $M D N 1$, corresponded to a region which has a stronger association 


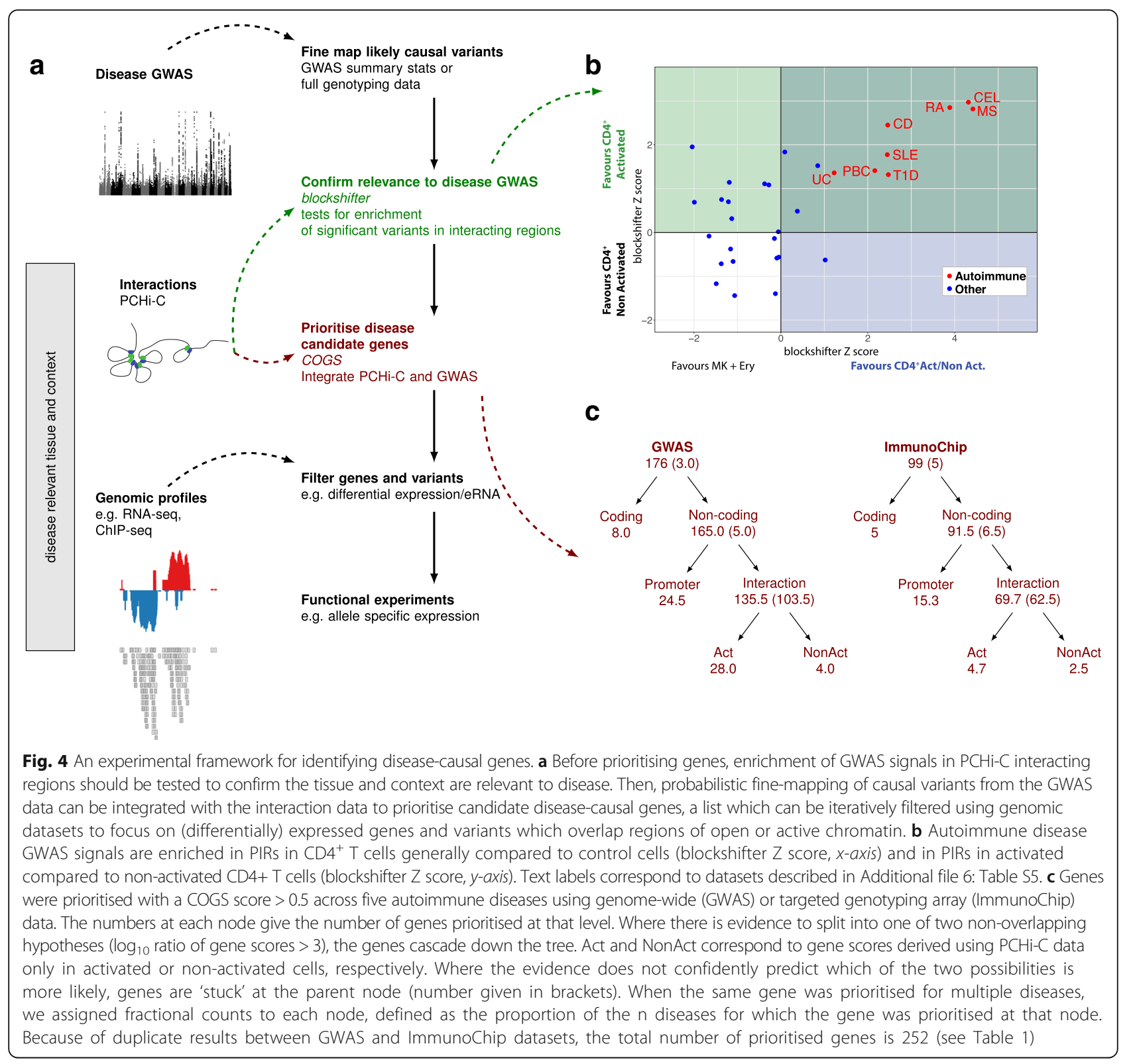

signal in the RA-ImmunoChip than RA-GWAS dataset, which may reflect the greater power of direct genotyping vs. imputation, given that the RA-ImmunoChip signal is echoed in ATD and T1D (Additional file 2: Figure S8). We prioritised a total of 245 unique protein-coding genes, 108 of which related to activation sensitive interactions (Additional files 9 and 10: Tables S7a and 7b, Fig. 4). Of 118 prioritised genes which could be related through interactions to a known susceptibility region, 63 (48\%) lay outside that disease susceptibility region. The median distance from peak signal to prioritised gene was $153 \mathrm{~kb}$. Note that prioritisation can be one (variant)-to-many (genes) because a single PIR can interact with more than one promoter and promoter fragments can contain more than one gene promoter. Note also that the score reflects both $\mathrm{PCHi}-\mathrm{C}$ interactions and the strength and shape of association signals (Additional file 2: Figure S9), therefore a subset of prioritised genes relate to an aggregation over sub-genomewide significant GWAS signals. This is therefore a 'long' list of prioritised genes which requires further filtering (Table 1). A total of 179 (of 245) prioritised genes were expressed in at least one activation state; we highlight specifically the subset of 118 expressed genes which can be related to a genome-wide significant GWAS signal through proximity of a genomewide significant SNP $\left(p<5 \times 10^{-8}\right)$ to a PIR. Of these, 82 were differentially expressed, 48 related to activationsensitive interactions and 63 showed overlap of GWAS fine-mapped variants with an expressed eRNA (Additional file 9: Table S7a). 
Table 1 Number of genes prioritised for autoimmune disease susceptibility under successive filters

\begin{tabular}{|c|c|c|}
\hline Group & Description & Number of genes \\
\hline 1 & Total & 245 \\
\hline 2 & ... Expressed & 179 \\
\hline 3 & $\begin{array}{l}\ldots \ldots \text { Proximal GWAS significant SNP } \\
\quad\left(p<5 \times 10^{-8}\right)\end{array}$ & 118 \\
\hline 4 & $\begin{array}{ccc}\ldots \ldots & \text { Prioritised gene differentially } \\
& \text { expressed upon activation }\end{array}$ & 82 \\
\hline 5 & $\begin{array}{c}\ldots \ldots \ldots \text { Prioritisation relates to activation } \\
\text { sensitive interactions }\end{array}$ & 48 \\
\hline 6 & $\begin{array}{c}\ldots \ldots \ldots \text { GWAS signal overlaps expressed } \\
\text { regRNA in at least one state }\end{array}$ & 63 \\
\hline
\end{tabular}

Note that group 2 is a subset of group 1, group 3 is a subset of group 2, and groups 4, 5 and 6 are all subsets of group 3 but not necessarily of each other

Taken together, our results reflect the complexity underlying gene regulation and the context-driven effects that common autoimmune disease-associated variants may have on candidate genes. Our findings are consistent with, and extend, previous observations $[7,8]$ and we highlight six examples which exemplify both activation-specific and activation-invariant interactions.

\section{Exemplar regions}

Here we highlight specific examples of prioritised genes with plausible autoimmune disease candidacy which illustrate three characteristics we found frequently, namely: (1) the identification of candidate genes some distance from association signals; (2) the tendency for multiple gene promoters to be identified as interacting with the same sets of disease-associated variants; and (3) genes prioritised in only one state of activation.

As an example of the first, CEL has been associated with a region on chromosome 1q31.2, for which RGS1 has been named as a causal candidate due to proximity of associated variants to its promoter [28]. Sub-genomewide significant signals for T1D (min. $p=1.5 \times 10^{-6}$ ) across the same SNPs which are associated with CEL have been interpreted as a co-localising T1D signal in the region [29]. RGS1 has recently been shown to have a role in the function of $\mathrm{T}$ follicular helper cells in mice [30], the frequencies of which and their associated IL-21 production have been shown to be increased in T1D patients [31]. However, our analysis also prioritises, in activated $\mathrm{T}$ cells, the strong functional candidate genes TROVE2 and UCHL5, over half a megabase distant and with three intervening genes not prioritised (Fig. 5).

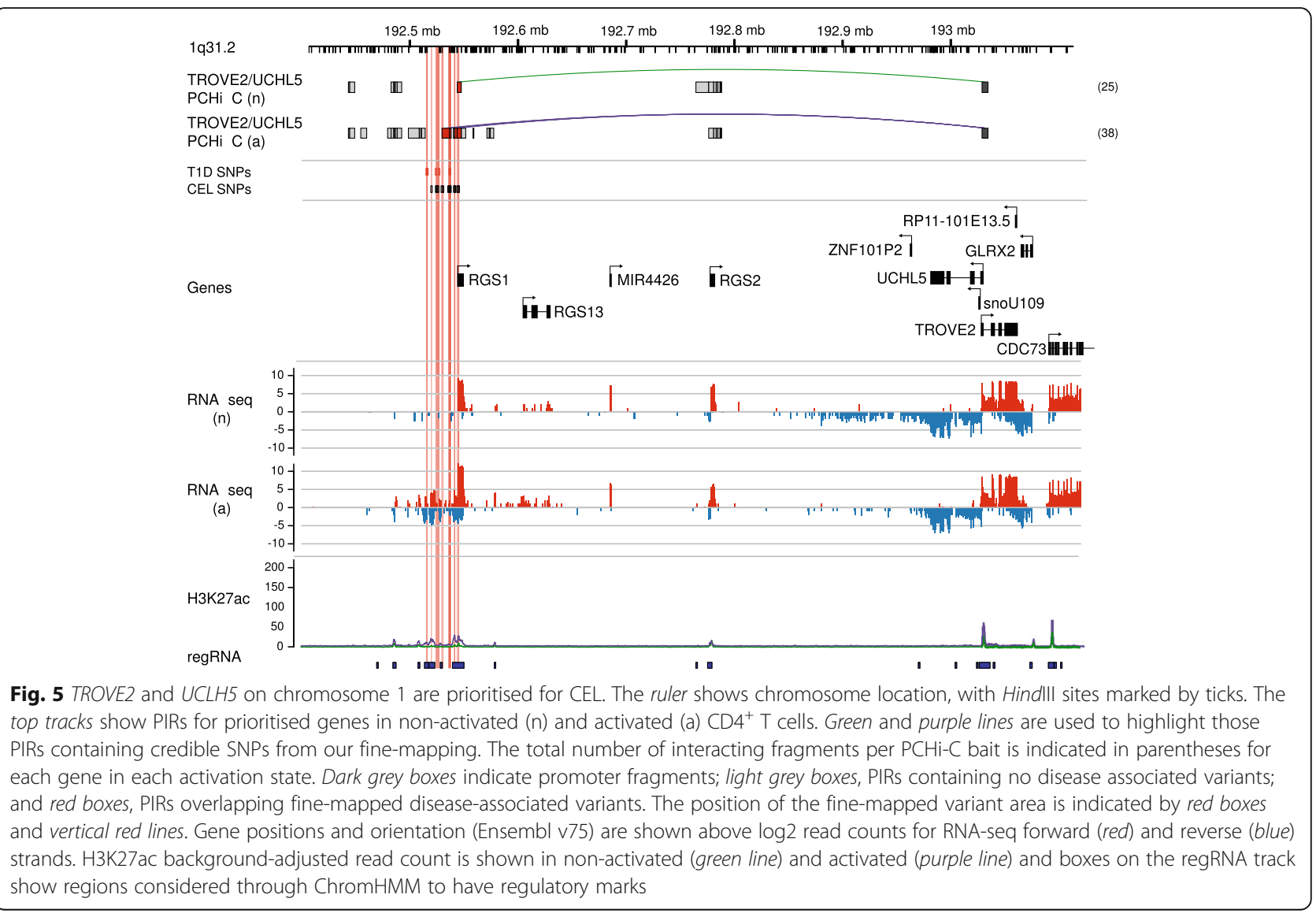


UCHL5 encodes ubiquitin carboxyl-terminal hydrolase-L5 a deubiquitinating enzyme that stabilises several Smad proteins and TGFBR1, key components of the TGF-beta1 signalling pathway $[32,33]$. TROVE2 is significantly upregulated upon activation $(\mathrm{FDR}=0.005)$ and encodes Ro60, an RNA binding protein that indirectly regulates type-I IFN-responses by controlling endogenous Alu RNA levels [34]. A global anti-inflammatory effect for TROVE2 expression would fit with its effects on gut (CEL) and pancreatic islets (T1D).

A similar situation is seen in the chromosome 1q32.1 region associated with T1D in which $I L 10$ has been named as a causal candidate gene [35]. Together with IL10, prioritised through proximity of credible SNPs to the IL10 promoter, we prioritised other IL10 gene family members IL19, IL20 and IL24 as well as two members of a conserved three-gene immunoglobulin-receptor cluster (FCMR and PIGR, Additional file 2: Figure S10). While IL19, IL20 and PIGR were not expressed in CD4 ${ }^{+}$T cells, FCMR was downregulated and IL24 and IL10 were upregulated following $\mathrm{CD}^{+} \mathrm{T}$ cell activation. IL-10 is recognised as an important anti-inflammatory cytokine in health and disease [36] and candidate genes FCMR and IL24 are components of a recently identified proinflammatory module in Th17 cells [37].

This region also exemplifies characteristic 2: a tendency for multiple gene promoters to be identified as interacting with the same sets of disease-associated variants. Parallel results have demonstrated co-regulation of multiple PCHi-C interacting genes by a single variant [37], suggesting that disease-related variants may act on multiple genes simultaneously, consistent with the finding that regulatory elements can interact with multiple promoters [38-40]. In this region, clusters of multiple adjacent PIRs were be detected for the same promoter fragments. It remains to be further validated whether all PIRs detected within such clusters correspond to 'causal' interactions or whether some such PIRs are 'bystanders' of strong interaction signals occurring in their vicinity. The use of PCHi$\mathrm{C}$ nonetheless adds considerable resolution compared to simply considering topologically associating domains (TADs), which have a median length of $415 \mathrm{~kb}$ in naive $\mathrm{CD}^{+}{ }^{+} \mathrm{T}$ cells [17] compared to a median of $37.5 \mathrm{~kb}$ total PIR length per gene in non-activated $\mathrm{CD} 4^{+} \mathrm{T}$ cells (Additional file 2: Figure S11).

Multiple neighbouring genes were also prioritised on chromosome 16q24.1: EMC8, COX4I1 and IRF8, the last only in activated $\mathrm{T}$ cells, for two diseases: RA and SLE (Additional file 2: Figure S12). Candidate causal variants for SLE and RA fine-mapped to distinct PIRs, yet all these PIRs interact with the same gene promoters, suggesting that interactions, possibly specific to different $\mathrm{CD}^{+} \mathrm{T}$ cell subsets, may allow us to unite discordant GWAS signals for related diseases [6, 41, 42]. EMC8 and
COX4I1 RNA expression was relatively unchanged by activation, whereas IRF8 expression was upregulated 97-fold, coincident with the induction of 16 intergenic IRF8 PIRs, four of which overlap autoimmune disease fine-mapped variants. Although the dominant effect of IRF8 is to control the maturation and function of the mononuclear phagocytic system [43], a $\mathrm{T}$ cell-intrinsic function regulating $\mathrm{CD} 4^{+}$Th17 differentiation has been proposed [44]. Our data further link the control of Th17 responses to susceptibility to autoimmune disease including RA and SLE [45].

Another notable example, $A H R$, was one of the 38 genes we prioritised in only one state of activation (characteristic 3, Fig. 4): for RA, $A H R$ was prioritised specifically in activated $\mathrm{T}$ cells rather than non-activated $\mathrm{T}$ cells (Additional file 2: Figure S13). $A H R$ is a high affinity receptor for toxins in cigarette smoke that has been linked to RA previously through differential expression in synovial fluid of patients, though not through GWAS [46]. Our analysis prioritises $A H R$ for RA due to a sub-genome-wide significant signal (rs71540792, $p=2.9 \times 10^{-7}$ ) and invites attempts to validate the genetic association in additional RA patients.

\section{Interaction-mediated regulation of IL2RA expression}

Given our prior interest in the potential for autoimmunedisease associated genetic variants to regulate IL2RA expression [42], we were interested to note $\mathrm{PCHi}-\mathrm{C}$ interactions between some of these variants and the IL2RA promoter. We attempted to confirm the predicted functional effects on $I L 2 R A$ expression experimentally. IL2RA encodes CD25, a component of the key trimeric cytokine receptor that is essential for high-affinity binding of IL-2, regulatory $\mathrm{T}$ cell survival and $\mathrm{T}$ effector cell differentiation and function [47]. Multiple variants in and near IL2RA have been associated with a number of autoimmune diseases $[35,48-50]$. We have previously fine-mapped genetic causal variants for T1D and multiple sclerosis (MS) in the IL2RA region [26], identifying five groups of SNPs in intron 1 and upstream of $I L 2 R A$, each of which is likely to contain a single disease-causal variant. Out of the group of eight SNPs previously denoted 'A' [26], three (rs12722508, rs7909519 and rs61839660) are located in an area of active chromatin in intron 1 , within a PIR that interacts with the IL2RA promoter in both activated and non-activated CD4 ${ }^{+}$ $\mathrm{T}$ cells (Fig. 6a). These three SNPs are also in LD with rs12722495 $\left(r^{2}>0.86\right)$ that has previously been associated with differential surface expression of CD25 on memory T cells [42] and differential responses to IL-2 in activated Tregs and memory $\mathrm{T}$ cells [51]. We measured the relative expression of IL2RA mRNA in five individuals heterozygous across all group A SNPs and homozygous across most other associated SNP groups, in a 4-h activation timecourse of $\mathrm{CD}^{+} \mathrm{T}$ cells. Allelic imbalance was observed 


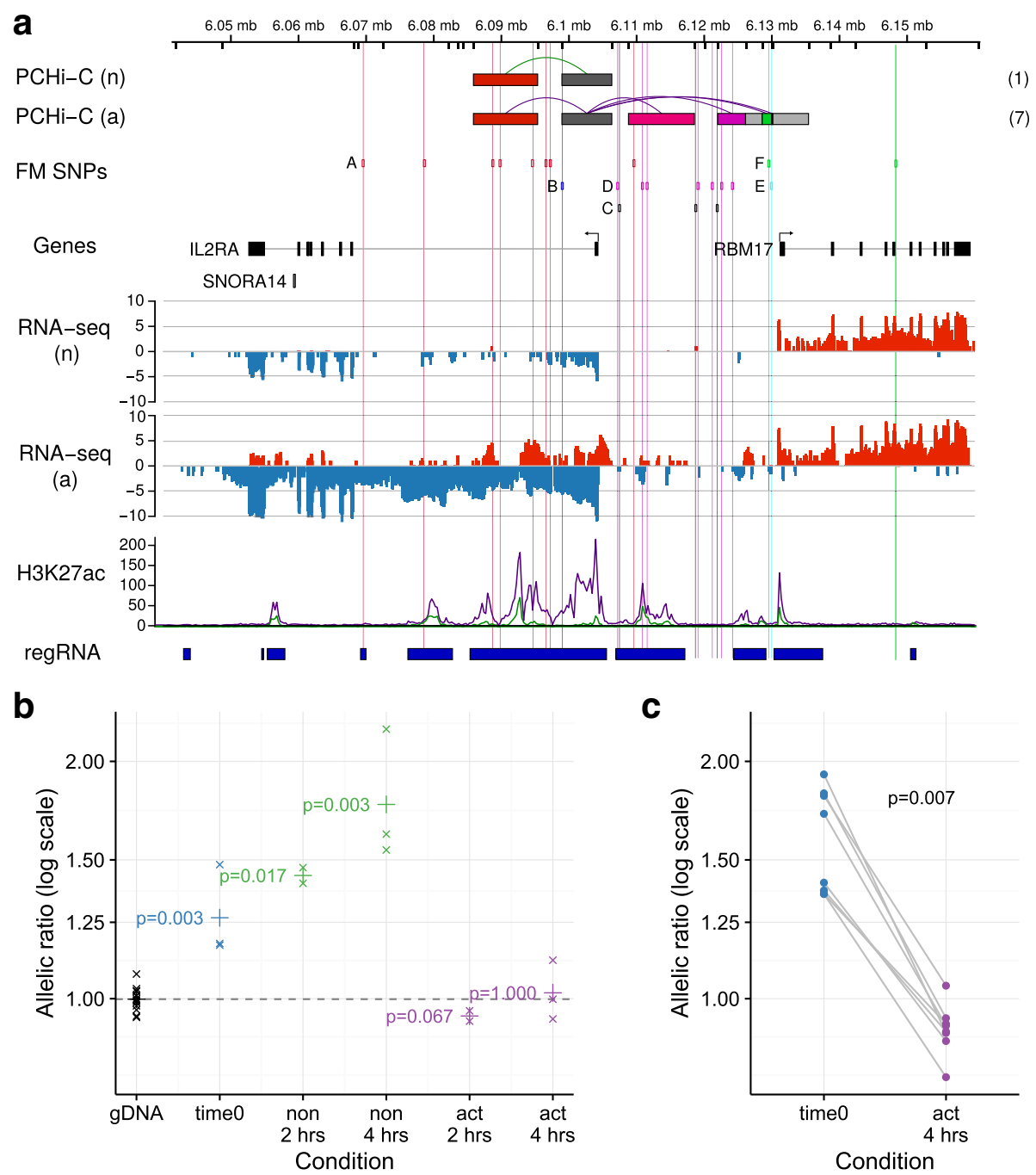

Fig. 6 PCHi-C interactions link the IL2RA promoter to autoimmune disease-associated genetic variation, which leads to expression differences in IL2RA mRNA. a The ruler shows chromosome location, with Hindlll sites marked by ticks. The top tracks show PIRs for prioritised genes in non-activated ( $\mathrm{n}$ ) and activated (a) CD4 ${ }^{+}$T cells. Green and purple lines are used to highlight those PIRs containing credible SNPs for the autoimmune diseases T1D and MS fine mapped on chromosome 10p15. Six groups of SNPs (A-F) highlighted in Wallace et al. [26] are shown, although note that group B was found unlikely to be causal. The total number of interacting fragments per $\mathrm{PCHi}-\mathrm{C}$ bait is indicated in parentheses for each gene in each activation state. Dark grey boxes indicate promoter fragments; light grey boxes, PIRs containing no disease-associated variants; and coloured boxes, PIRs overlapping fine-mapped disease-associated variants. PCHi-C interactions link a region overlapping group A in non-activated and activated CD4+ T cells to the IL2RA promoter (dark grey box) and regions overlapping groups D and F in activated CD4+ T cells only. RNA-seq reads (log 2 scale, red $=$ forward strand, blue = reverse strand) highlight the upregulation of IL2RA expression upon activation and concomitant increases in H3K27ac (non-activated, n, green line; activated, a, purple line) in the regions linked to the IL2RA promoter. Red vertical lines mark the positions of the group A SNPs. Numbers in parentheses show the total number of IL2RA PIRs detected in each state. Here we show those PIRs proximal to the IL2RA promoter. Comprehensive interaction data can be viewed at https://www.chicp.org. b Allelic imbalance in mRNA expression in total CD4+ T cells from individuals heterozygous for group A SNPs using rs12722495 as a reporter SNP in non-activated (non) and activated (act) CD4 ${ }^{+}$T cells cultured for 2 or $4 \mathrm{~h}$, compared to genomic DNA (gDNA, expected ratio $=1$ ). Allelic ratio is defined as the ratio of counts of $\mathrm{T}$ to $\mathrm{C}$ alleles. ' $\mathrm{X}$ ' $=$ geometric mean of the allelic ratio over 2-3 replicates within each of $4-5$ individuals; $p$ values from a Wilcoxon rank sum test comparing complementary DNA (cDNA) to gDNA are shown. ' + ' shows the geometric mean allelic ratio over all individuals. $c$ Allelic imbalance in mRNA expression in memory CD4 ${ }^{+} T$ cells differs between ex vivo (time 0 ) and 4 -h activated samples from eight individuals heterozygous for group A SNPs using rs12722495 as a reporter SNP. $p$ value from a paired Wilcoxon signed rank test is shown

consistently for two reporter SNPs in intron 1 and in the 3' UTR in non-activated $\mathrm{CD}^{+} \mathrm{T}$ cells in each individual (Fig. 6b; Additional file 2: Figure S14a), validating a functional effect of the $\mathrm{PCHi}-\mathrm{C}$-derived interaction between this
PIR and the IL2RA promoter in non-activated $\mathrm{CD}^{+}{ }^{+} \mathrm{T}$ cells. While the allelic imbalance was maintained in nonactivated cells cultured for 2-4 h, the imbalance was lost in cells activated under our in vitro conditions. Since 
increased CD25 expression with rare alleles at group A SNPs has previously been observed on memory $\mathrm{CD} 4^{+} \mathrm{T}$ cells but not the naive or Treg subsets that are also present in the total $\mathrm{CD}_{4}^{+} \mathrm{T}$ cell population [42], we purified memory cells from eight group A heterozygous individuals and confirmed activation-induced loss of allelic imbalance of IL2RA mRNA expression in this more homogeneous population (Fig. 6c, Additional file 2: Figure S14b; Wilcoxon $p=0.007$ ). IL2RA is one of the most strongly upregulated genes upon $\mathrm{CD} 4^{+} \mathrm{T}$ cell activation, showing a 65-fold change in expression in our RNA-seq data. Concordant with the genome-wide pattern (Fig. 3), the IL2RA promoter fragment gains PIRs that accumulate H3K27ac modifications upon activation and these, as well as potentially other changes marked by an increase in H3K27ac modification at rs61839660 and across the group A SNPs in intron 1, could account for the loss of allelic imbalance. These results emphasise the importance of steadystate CD25 levels on $\mathrm{CD}_{4}^{+} \mathrm{T}$ cells for the disease association mediated by the group A SNPs, levels which will make the different subsets of $\mathrm{CD} 4^{+} \mathrm{T}$ cells more or less sensitive to the differentiation and activation events caused by IL-2 exposure in vivo [52].

\section{Discussion}

Our results illustrate the changes in chromosome conformation detected by $\mathrm{PCHi}-\mathrm{C}$ in a single cell type in response to a single activation condition. That the $\mathrm{PCHi}-\mathrm{C}$ technique can indeed link enhancers to their target genes is supported by our evidence that the direction of fold changes at eRNAs is connected to those at their PCHi-C linked protein-coding genes. Our results also provide support for the candidacy of certain genes and sequences in GWAS regions as causal for disease. Recent attempts to link GWAS signals to variation in gene expression in primary human cells have sometimes found only limited overlap [53-55]. One explanation may be that these experiments miss effects in specific cell subsets or states, especially given the transcriptional diversity between the many subsets of memory $\mathrm{CD} 4^{+} \mathrm{T}$ cells [56]. We highlight the complex nature of disease association at the IL2RA region where additional PIRs for IL2RA gained upon activation overlap other fine-mapped disease-causal variants (Fig. 6a), suggesting that other allelically imbalanced states may exist in activated cells, which may also correspond to altered disease risk. For example, the PIR containing rs61839660, a group A SNP, also contains an activation expression quantitative trait locus (eQTL) for IL2RA expression in $\mathrm{CD}^{+} \mathrm{T}$ effectors [57] marked by rs12251836, which is unlinked to the group A variants and was not associated with T1D [57]. Furthermore, rs61839660 itself has recently been reported as a QTL for methylation of the IL2RA promoter as well as an eQTL for IL2RA expression in whole blood $[55,58]$. The differences between
CD25 expression in different $\mathrm{T}$ cell subsets $[59,60]$ and the rapid activation-induced changes in gene and regulatory expression, chromatin marks and chromosome interactions we observe, imply that a large diversity of cell types and states will need to be assayed to fully understand the identity and effects of autoimmune diseasecausal variants.

Our approach, like others such as eQTL analyses and integration of GWAS variants with chromatin state information, offers a view of disease through the prism of purified cell subsets in specific states of activation. However, a more complete range of cell types and activation states will be needed for the comprehensive understanding of complex diseases for which multiple cell types are aetiologically involved. It will be challenging to assay this greater diversity of cell types and states in the large numbers of individuals needed for traditional eQTL studies, particularly for cell-type or condition-specific eQTLs that have been shown to generally have weaker effects $[61,62]$. Allele-specific expression (ASE) is a more powerful design to quantify the effects of genetic variation on gene expression with modest sample sizes [63] and the targeted ASE approach that we adopt enables testing of individual variants or haplotypes at which donors are selected to be heterozygous, while controlling for other potentially related variants at which donors are selected to be homozygous.

\section{Conclusions}

Here we have presented an approach for connecting disease-associated variants derived from GWAS with putative target genes based on promoter interactome maps obtained with $\mathrm{PCHi}-\mathrm{C}$. By using statistical finemapping of GWAS data, integrated with $\mathrm{PCHi}-\mathrm{C}$, to highlight both likely disease-causal variants and their potential target genes, we enable the design of targeted ASE analyses for functional confirmation of individual effects. This systematic experimental framework offers an alternative approach to candidate causal gene identification for variants with cell state-specific functional effects, with achievable sample sizes.

\section{Methods \\ $\mathrm{CD} 4^{+} \mathrm{T}$ cell purification and activation, preparation for genomics assays}

Blood samples were obtained from healthy donors selected from the Cambridge BioResource. Donors were excluded if they were diagnosed with autoimmune disease or cancer, were receiving immunosuppressants, cytotoxic agents or intravenous immunoglobulin or had been vaccinated or received antibiotics in the 2-4 weeks preceding the blood donation. $\mathrm{CD}^{+} \mathrm{T}$ cells were isolated from whole blood using RosetteSep (STEMCELL technologies, Canada) according to the manufacturer's 
instructions. Purified $\mathrm{CD}^{+} \mathrm{T}$ cells (average, 96.5\% pure; range, 92.9-98.7\%) were washed in X-VIVO 15 supplemented with $1 \%$ AB serum (Lonza, Switzerland) and penicillin/streptomycin (Invitrogen, UK) and plated in 96-well CELLSTAR U-bottomed plates (Greiner Bio-One, Austria) at a concentration of $2.5 \times 10^{5}$ cells/well. Cells were left untreated or stimulated with Dynabeads human $\mathrm{T}$ activator $\mathrm{CD} 3 / \mathrm{CD} 28$ beads (Invitrogen, $\mathrm{UK}$ ) at a ratio of 1 bead : 3 cells for $4 \mathrm{~h}$ at $37{ }^{\circ} \mathrm{C}$ and $5 \% \mathrm{CO}_{2}$. Cells were harvested, centrifuged, supernatant removed and either: (1) resuspended in RLT buffer (RNeasy micro kit, Qiagen, Germany) for RNA-seq $\left(0.75-1 \times 10^{6} \mathrm{CD}^{+} \mathrm{T}\right.$ cells/pool and activation state); or (2) fixed in formaldehyde for capture $\mathrm{Hi}-\mathrm{C}\left(44-101 \times 10^{6} \mathrm{CD}^{+} \mathrm{T}\right.$ cells/pool and activation state) or ChIP-seq $\left(16-26 \times 10^{6} \mathrm{CD}^{+} \mathrm{T}\right.$ cells/pool and activation state) as detailed in [17].

ChIP-seq (H3K27ac, H3K4me1, H3K4me3, H3K27me3, H3K9me3, H3K36me3) was carried out according to BLUEPRINT protocols [17]. Formaldehyde fixed cells were lysed, sheared and DNA sonicated using a Bioruptor Pico (Diagenode). Sonicated DNA was pre-cleared (Dynabeads Protein A, Thermo Fisher) and ChIP performed using BLUEPRINT validated antibodies and the IP-Star automated platform (Diagenode). Libraries were prepared and indexed using the iDeal library preparation kit (Diagenode) and sequenced (Illumina HiSeq, paired-end).

For PCHi-C [17], DNA was digested overnight with HindIII, end-labelled with biotin-14-dATP and ligated in preserved nuclei. De-crosslinked DNA was sheared to an average size of $400 \mathrm{bp}$, end-repaired and adenine-tailed. Following size selection (250-550 bp fragments), biotinylated ligation fragments were immobilised, ligated to paired-end adaptors and libraries amplified (7-8 polymerase chain reaction $[\mathrm{PCR}]$ amplification rounds). Biotinylated 120-mer RNA baits targeting both ends of HindIII restriction fragments that overlap Ensemblannotated promoters within the Ensembl categories of protein-coding, non-coding, antisense, snRNA, miRNA and snoRNA were used to capture targets [64]. After enrichment, the library was further amplified (four PCR cycles) and sequenced on the Illumina HiSeq 2500 platform. Each PCHi-C library was sequenced over three lanes generating 50-bp paired-end reads.

\section{$\mathrm{PCHi}-\mathrm{C}$ interaction calls}

Raw sequencing reads were processed using the HiCUP pipeline [65] and interaction confidence scores were computed using the CHiCAGO pipeline [14] as previously described [17]. We considered the set of interactions with high confidence scores $(>5)$ in this paper.

Raw PCHi-C read counts from three replicates and two conditions were transformed into a matrix and a trimmed mean of M-values normalisation was applied to account for library size differences. Subsequently, a voom normalisation was applied to log-transformed counts in order to estimate precision weights per contact and differential interaction estimates were obtained after fitting a linear model on a paired design, using the limma Bioconductor $R$ package [66].

\section{Microarray measurement of gene expression}

We recruited 20 healthy volunteers from the Cambridge BioResource. Total $\mathrm{CD}_{4}^{+} \mathrm{T}$ cells were isolated from whole blood within $2 \mathrm{~h}$ of venepuncture by RosetteSep (StemCell Technologies). To assess the transcriptional variation in response to TCR stimulation, $10^{6} \mathrm{CD}^{+} \mathrm{T}$ cells were cultured in U-bottom 96-well plates in the presence or absence of human $\mathrm{T}$ activator CD3/CD28 beads at a ratio of 1 bead : 3 cells. Cells were harvested at $2,4,6$ or $21 \mathrm{~h}$ post stimulation or after 0,6 or $21 \mathrm{~h}$ in the absence of stimulation. Three samples from the 6-h unstimulated time point were omitted from the study due to insufficient cell numbers and a further four samples were dropped after quality control, resulting in a total of 133 samples that were included in the final analysis. RNA was isolated using the RNAeasy kit (Qiagen) according to the manufacturer's instructions.

cDNA libraries were synthesised from $200 \mathrm{ng}$ total RNA using a whole-transcript expression kit (Ambion) according to the manufacturer's instructions and hybridised to Human Gene 1.1 ST arrays (Affymetrix). Microarray data were normalised using a variance stabilising transformation [67] and differential expression was analysed in a paired design using limma [66]. Genes were clustered into modules using WGCNA [68].

\section{ChIP-sequencing and regulatory annotation}

ChIP-seq reads for all histone modification assays and control experiments were mapped to the reference genome using BWA-MEM [69], a Burrows-Wheeler genome aligner. Samtools [70] was employed to filter secondary and low-quality alignments (we retained all read pair alignments with PHRED score $>40$ that matched all bits in SAM octal flag 3 and did not match any bits in SAM octal flag 3840). The remaining alignments were sorted, indexed and a whole-genome pileup was produced for each histone modification, sample and condition triple.

We used ChromHMM [20], a multivariate hidden Markov model, to perform a whole-genome segmentation of chromatin states for each activation condition. First, we binarised read pileups for each chromatin mark pileup using the corresponding control experiment as a background model. Second, we estimated the parameters of a 15-state hidden Markov model (a larger state model resulted in redundant states) using chromosome 1 data 
from both conditions. Parameter learning was re-run five times using different random seeds to assess convergence. Third, a whole-genome segmentation was produced for each condition by running the obtained model on the remaining chromosomes. Each state from the obtained model was manually annotated and states indicating the presence of promoter or enhancer chromatin tags were selected (E4-E11, Additional file 2: Figure S6). Overlapping promoter or enhancer regions in nonactivated and activated genome segmentations were merged to create a $\mathrm{CD} 4^{+} \mathrm{T}$ cell regulatory annotation. Thus, we defined 53,534 regulatory regions (Additional files 11, 12 and 13: Tables S8a-S8c).

\section{RNA sequencing}

Total RNA was isolated using the RNeasy kit (Qiagen) and the concentrations and integrity were quantified using Bioanalyzer (Agilent); all samples reached RINs $>9.8$. Two pools of RNA were generated from three and four donors and for each experimental condition. cDNA libraries were prepared from 1 ug total RNA using the stranded NEBNext Ultra Directional RNA kit (New England Biolabs) and sequenced on HiSeq (Illumina) at an average coverage of 38 million paired-end reads/pool. RNA-seq reads were trimmed to remove traces of library adapters by matching each read with a library of contaminants using Cutadapt [71], a semi-global alignment algorithm. Owing to our interest in detecting functional enhancers, which constitute transcription units on their own right, we mapped reads to the human genome using STAR [72], a splicingaware aligner. This frees us from relying on a transcriptome annotation which would require exact boundaries and strand information for all features of interest, something not available in case of promoters and enhancers.

After alignment, we employed Samtools [70] to discard reads with an unmapped pair, secondary alignments and low-quality alignments. The resulting read dataset, with an average of 33 million paired-end reads/sample, was sorted and indexed. We used FastQC (v0.11.3, http:// www.bioinformatics.babraham.ac.uk/projects/fastqc/) to ensure all samples had regular GC content (sum of deviations from normal includes less than $15 \%$ of reads), base content per position (difference A vs. T and G vs. C less than $10 \%$ at all positions) and kmer counts (no imbalance of kmers with $p<0.01$ ) as defined by the tool. We augmented Ensembl 75 gene annotations with regulatory region definitions obtained from our ChIP-seq analysis described above and defined them as present in both genome strands due to their bi-directional transcription potential. For each RNA-seq sample, we quantified expression of genomic and regulatory features in a two-step strand-aware process using HTSeq. [73] For each gene we counted the number of reads that fell exactly within its exonic regions and did not map to other genomic elements. For each regulatory feature, we counted the number of reads that fell exactly within its defined boundaries and did not map to other genomic or regulatory elements.

By construction, this quantification scheme counts each read at most once towards at most one feature. Furthermore, strand information is essential to be able to assign reads to features in regions with overlapping annotations. For example, distinguishing intronic eRNAs from pre-mRNA requires reads originating from regulatory activity in the opposite strand from the gene.

Feature counts were transformed into a matrix and a trimmed mean of $\mathrm{M}$-values normalisation was applied to account for library size differences, plus a filter to discard features below an expression threshold of $<0.4$ counts per million mapped reads in at least two samples, a rather low cutoff, to allow for regulatory RNAs to enter differential expression calculations. This threshold equates to approximately 15 reads, given our mapped library sizes of $\sim 35$ million paired-end reads. A voom normalisation was applied to log-transformed counts in order to estimate precision weights per gene and differential expression estimates were obtained after fitting a linear model on a paired design, using the limma Bioconductor $\mathrm{R}$ package [66]. There was a strong correlation (rho $=0.81$ ) between microarray and RNA-seq fold change estimates at $4 \mathrm{~h}$.

\section{Comparison of regRNAs to FANTOM CAGE data}

We compared expressed regRNA regions detected in our non-activated $\mathrm{CD}^{+} \mathrm{T}$ cell samples vs. those found using CAGE-seq by the FANTOM5 Consortium. RNA-seq, using a regulatory reference obtained from chromatin states, yields 17,175 features expressed with at least 0.4 counts per million in both non-activated $\mathrm{CD} 4^{+} \mathrm{T}$ cell samples. Among those, 3897 correspond to regulatory regions. Unstimulated $\mathrm{CD}^{+}$samples from FANTOM5 (http://fantom.gsc.riken.jp/5/datafiles/latest/basic/human.primary_cell.hCAGE/, samples 10,853, 11,955 and 11998) contain 266,710 loci expressed (with at least one read) in all three samples.

We found 13,178 of our 17,175 expressed CD4 $4^{+} \mathrm{T}$ cell features overlap expressed loci in CAGE data (77\%). Conversely, 243,596/266,710 CAGE loci overlap $\mathrm{CD}^{+}{ }^{+} \mathrm{T}$ cell features (91\%). Similarly, 2888/3897 expressed regRNAs overlap expressed loci in CAGE data (74\%).

\section{Comparison of $\mathrm{PCH}-\mathrm{C}$ and $\mathrm{ChIA-PET}$ interactions}

We downloaded supplementary Table 1 from http:// www.nature.com/cr/journal/v22/n3/extref/cr201215x1.xlsx [15] and counted the overlaps of $\mathrm{PCHi}-\mathrm{C}$ interactions from $\mathrm{CD} 4^{+} \mathrm{T}$ cells and comparitor cells (megakaryoctyes and erythroblasts) in distance bins. $\mathrm{R}$ code to replicate the analysis is at https://github.com/chr1swallace/cd4-pchic/ 
blob/master/analyses/chepelev-comparison.R. Calling interactions requires correction for the expected higher density of random collisions at shorter distances [74] which are explicitly modelled by CHICAGO [14] used in this study but not in the ChIA-PET study [15]. As a result, we expected a higher false-positive rate from the ChIA-PET data at shorter distances.

\section{Regression of gene expression against PIR count and eRNA expression}

We related measures of gene expression (absolute log2 counts or $\log 2$ fold change) to numbers of PIRs or numbers of PIRs overlapping specific features using linear regression. We used logistic regression to relate agreement between fold change direction at PCHi-C linked protein-coding genes and eRNAs. We used robust clustered variance estimates to account for the shared baits for some interactions across genes with the same prey. Enrichment of chromatin marks in interacting baits and prey were assessed by logistic regression modelling of a binary outcome variable (fragment overlapped specific chromatin peak) against a fragment width and a categorical explanatory variable (whether the HindIII fragment was a bait or prey and the cell state the interaction was identified in), using block bootstrapping of baited fragments (https://github.com/chr1swallace/genomic.autocorr) to account for spatial correlation between neighbouring fragments.

\section{GWAS summary statistics}

We used a compendium of 31 GWAS datasets [17] (Additional file 6: Table S5). Briefly, we downloaded publicly available GWAS statistics for 31 traits. Where necessary we used the liftOver utility to convert these to GRCh37 coordinates. To remove spurious association signals, we removed variants with $p<5 \times 10^{-8}$ for which there were no variants in $\operatorname{LD}\left(\mathrm{r}^{2}>0.6\right.$ using 1000 genomes EUR cohort as a reference genotype panel) or within $50 \mathrm{~kb}$ with $p<10^{-5}$. We masked the MHC region (GRCh37:chr6:25-35 Mb) from all downstream analysis due to its extended LD and known strong and complex associations with autoimmune diseases.

Comparison of GWAS data and PIRs requires dense genotyping coverage. For GWAS which did not include summary statistics imputed for non-genotyped SNPs, we used a poor man's imputation (PMI) method [17] to impute. We imputed $p$ values at ungenotyped variants from 1000 Genomes EUR phase 3 by replacing missing values with those of their nearest proxy variant with $r^{2}>0.6$, if one existed. Variants that were included in the study but did not map to the reference genotype set were also discarded.

To calculate posterior probabilities that each SNP is causal under a single causal variant assumption, we divided the genome into linkage disequilibrium blocks of $1 \mathrm{cM}$ based on data from the HapMap project (ftp:// ftp.ncbi.nlm.nih.gov/hapmap/recombination/2011-01_phaseII_B37/). For each region excluding the $\mathrm{MHC}$ we used code modified from Giambartolomei et al. [75] to compute approximate Bayes factors for each variant using the Wakefield approximation [76]; thus, posterior probabilities that each variant was causal as previously proposed [77]. The method assumes a normal prior on the population log relative risk centred at 0 and we set the variance of this distribution to 0.04 , equivalent to a $95 \%$ belief that the true relative risk is in the range of $0.66-$ 1.5 at any causal variant. We set the prior probability that any variant is causal for disease to $10^{-4}$.

\section{Testing of the enrichment of GWAS summary statistics in PIRs using blockshifter}

We used the blockshifter method [17] (https:/github.com/ ollyburren/CHIGP) to test for a difference between variant posterior probability distributions in HindIII fragments with interactions identified in test and control cell types using the mean posterior probability as a measure of central location. Blockshifter controls for correlation within the GWAS data due to LD and interaction restriction fragment block structure by employing a rotating label technique similar to that described in GoShifter [25] to generate an empirical distribution of the difference in means under the null hypothesis of equal means in the test and control set. Runs of one or more PIRs (separated by at most one HindIII fragment) are combined into 'blocks', that are labelled unmixed (either test or control PIRs) or mixed (block contains both test and control PIRs). Unmixed blocks are permuted in a standard fashion by reassigning either test or control labels randomly, taking into account the number of blocks in the observed sets. Mixed blocks are permuted by conceptually circularising each block and rotating the labels. A key parameter is the gap size-the number of non-interacting HindIII fragments allowed within a single block, with larger gaps allowing for more extended correlation.

We used simulation to characterise the type 1 error and power of blockshifter under different conditions and to select an optimal gap size. First, from the Javierre et al. dataset [17], we selected a test (Activated or Non Activated $\mathrm{CD}^{+}{ }^{+} \mathrm{T}$ Cells) and control (Megakaryocyte or Erythroblast) set of PIRs with a CHiCAGO score $>5$, as a reference set for blockshifter input.

Using the European 1000 genomes reference panel, we simulated GWAS summary statistics, under different scenarios of GWAS/PIR enrichment. We split chromosome 1 into $1 \mathrm{cM}$ LD blocks and used reference genotypes to compute a covariance matrix for variants with minor allele frequency above $1 \%, \Sigma$. GWAS Z scores can be simulated as multivariate 
normal with mean $\mu$ and variance $\Sigma$ [78]. Each block may contain no causal variants $\left(G_{W A S}\right.$ null,$\left.\mu=0\right)$ or one $\left(\mathrm{GWAS}_{\text {alt }}\right)$. For $\mathrm{GWAS}_{\text {alt }}$ blocks, we pick a single causal variant, $i$, and calculate the expected noncentrality parameter (NCP) for a 1 degree of freedom chi-square test of association at this variant and its neighbours. This framework is natural because, under a single causal variant assumption, the NCP at any variant $j$ can be expressed as the NCP at the causal variant multiplied by the $\mathrm{r}^{2}$ between variants $i$ and $j$ [79]. In each case, we set the NCP at the causal variant to 80 to ensure that each causal variant was genome-wide significant $(p<$ $\left.5 \times 10^{-8}\right) . \mu$ is defined as the square root of this constructed NCP vector.

For all scenarios, we randomly chose $50 \mathrm{GWAS}_{\text {alt }}$ blocks leaving the remaining 219 GWAS $_{\text {null }}$. Enrichment is determined by the preferential location of simulated causal variants within test PIRs. In all scenarios, each causal variant has a $50 \%$ chance of lying within a PIR, to mirror a real GWAS in which we expect only a proportion of causal variants to be regulatory in any given cell type. Under the enrichment-null scenario, used to confirm control of type 1 error rate, the remaining variants were assigned to PIRs without regard for whether they were identified in test or control tissues. To examine power, we considered two different scenarios with PIR-localised causal variants chosen to be located specifically in test PIRs with either $50 \%$ probability, scenario power (1) or $100 \%$, scenario power (2). Note that a PIR from the test set may also be in the control set, thus, as with a real GWAS, not all causal variants will be informative for this test of enrichment.

For each scenario, we further considered variable levels of genotyping density, corresponding to full genotyping (everything in 1000 Genomes), HapMap imputation (the subset of SNPs also in Stahl et al. [80] dataset) or genotyping array (the subset of SNPs also on the Illumina $550 \mathrm{k}$ array). Where genotyping density is less than full, we used our proposed PMI strategy to fill in $\mathrm{Z}$ scores for missing SNPs.

We ran blockshifter, with 1000 null permutations, for each scenario and PMI condition for 4000 simulated GWAS, with a blockshifter superblock gap size parameter (the number of contiguous non-PIR HindIII fragments allowed within one superblock) of between 1 and 20 and supplying numbers of cases and controls from the RA dataset [49].

For comparison, we also investigated the behaviour of a naive test for enrichment for the null scenario. We computed a $2 \times 2$ table variants according to test and control PIR overlap, and whether a variant's posterior probability of causality exceeded an arbitrary threshold of 0.01 , and Fisher's exact test to test for enrichment.

\section{Enrichment of GWAS summary statistics in $\mathrm{CD}^{+}$and activated $\mathrm{CD}^{+}$PIRs}

We compared the following sets using all GWAS summary statistics, with a superblock gap size of 5 (obtained from simulations above) and 10,000 permutations under the null:

- Total $\mathrm{CD}^{+}$Activated + Total $\mathrm{CD} 4^{+}$NonActivated (test) vs. endothelial precursors + megakaryocytes (control)

- Total $\mathrm{CD} 4^{+}$Activated (test) vs. Total $\mathrm{CD} 4^{+}$NonActivated (control)

\section{Variant posterior probabilities of inclusion, full genotype} data (ImmunoChip)

We carried out formal imputation to 1000 Genomes Project EUR data using IMPUTE2 [81] and fine-mapped causal variants in each of the 179 regions where a minimum $p<0.0001$ was observed using a stochastic search method which allows for multiple causal variants in a region (https://github.com/chr1swallace/GUESSFM) [26]. Despite the pre-selection of regions associating with autoimmune diseases on the ImmunoChip, we chose to again set the prior probability that any variant was causal to $10^{-4}$, to align our analysis with that applied to the GWAS summary data. The prior probability for individual models follows a binomial distribution, according to the number of causal variants represented, so that the prior for each of the $\left(\begin{array}{l}n \\ k\end{array}\right) k$ - SNP causal variant models was $\left(10^{-4}\right)^{k}\left(1-10^{-4}\right)^{(n-k)}$ where $n$ is the number of SNPs considered in the region. The posterior probabilities for models that contained variants which overlapped PIRs for each gene were aggregated to compute PIR-level marginal posterior probabilities of inclusion.

\section{Variant posterior probabilities of inclusion, summary statistics}

Where we have only summary statistics of GWAS data already imputed to 1000 Genomes, we divided the genome into linkage disequilibrium blocks of $0.1 \mathrm{cM}$ based on data from the HapMap project (ftp://ftp.ncbi.nlm.nih.gov/hapmap/recombination/2011-01_phaseII_B37/). For each region, excluding the MHC, we use code modified from Giambartolomei et al. [75] to compute approximate Bayes factors for each variant using the Wakefield approximation [76]; thus, posterior probabilities that each variant was causal assuming at most one causal variant per region as previously proposed [77].

\section{Computation of gene prioritisation scores}

We used the COGS method [17] (https://github.com/ollyburren/CHIGP) to prioritise genes for further analysis. 
We assign variants to the first of the following three categories it overlaps for each annotated gene, if any:

1. coding variant: the variant overlaps the location of a coding variant for the target gene;

2. promoter variant: the variant lies in a region baited for the target gene or adjacent restriction fragment;

3. PIR variant: the variant lies in a region overlapping any PIR interacting with the target gene.

We produced combined gene/category scores by aggregating, within LD blocks, over models with a variant in a given set of PIRs (interacting regions) or over HindIII fragments baited for the gene promoter and immediate neighbours (promoter regions) or over coding variants to generate marginal probabilities of inclusion (MPPI) for each hypothesised group. We combine these probabilities across LD blocks, $i$, using standard rules of probability to approximate the posterior probability that at least one LD block contains a causal variant:

$$
\text { gene score }=1-\prod_{i \in \text { LD blocks }}(1-[\text { score for } i])
$$

Thus, the score takes a value between 0 and 1 , with 1 indicating the strongest support. We report all results with score $>0.01$ in Additional file 9: Table S7b, but focus in this manuscript on the subset with scores $>0.5$.

Because COGS aggregates over multiple signals, a gene may be prioritised because of many weak signals or few strong signals in interacting regions. To estimate the expected number of prioritised genes for a typical GWAS signal, we considered the subset of 76 input regions with genome-wide significant signals $\left(p<5 \times 10^{-8}\right)$ in ImmunoChip datasets. We prioritised at least one gene with a COGS score $>0.5$ in 35 regions, with a median of three genes/region (interquartile range $[\mathrm{IQR}]=1.5-4$ ). Equivalent analysis of the genome-wide significant GWAS signals prioritised a median of two genes/region (IQR $=1-3$ ). This suggests that this algorithm might be expected to prioritise at least one gene in about half the genome-wide significant regions input when run on a relevant cell type.

We developed a hierarchical heuristic method to ascertain for each target gene, which was the mostly likely component and cell state. First, for each gene we compute the gene score due to genic variants (components $1+2$ ) and variants in PIRs (component 3) using all available tissue interactions for that gene. While components 1 and 2 are fixed for a given gene and trait, the contribution of variants overlapping PIRs varies depending on the tissue context being examined. We use the ratio of the genic score to PIRs score in a similar manner to a
Bayes factor to decide whether causal variants contributing to the gene score are more likely to lie within the gene or within its associated PIRs. If a genic location is more likely (gene.score ratio $>3$ ) we iterate and compare if the gene score due to coding variants (component 1) is more likely than for promoter variants (component 2 ). Similarly, if PIRs are more likely, we compare PIR gene scores for activated vs. non-activated cells. If at any stage no branch is substantially preferred over its competitor (ratio of gene scores $<3$ ), we return the previous set as most likely, otherwise we continue until a single cell state/set is chosen. In this way, we can prioritise genes based on the overall score and label as to a likely mechanism for candidate causal variants.

\section{Allele-specific expression assays}

Total $\mathrm{CD} 4^{+} \mathrm{T}$ cells were isolated from five healthy donors and activated as described above and were harvested after 0,2 and $4 \mathrm{~h}$ in RLT Plus buffer. Selected donors were heterozygous for all eight group A SNPs and homozygous for group C and F SNPs. Two and three of the donors were homozygous for the group D and E SNP groups, respectively (Additional file 14: Table S9). Memory $\mathrm{CD}^{+} \mathrm{T}$ cells were sorted from cryopreserved PBMC from an additional eight healthy donors as viable, $\alpha \beta \mathrm{TCR}^{+}, \mathrm{CD}^{+}, \mathrm{CD} 45 \mathrm{RA}^{-}$, $\mathrm{CD} 127^{+}$and $\mathrm{CD} 27^{+}$cells using a FACSAria III cell sorter (BD Biosciences). Sorted cells were either activated for $4 \mathrm{~h}$ in culture as described above or resuspended directly in RLT plus buffer post-sort. Total RNA was extracted using Qiagen RNeasy Micro plus kit and cDNA was synthesised using Superscript III reverse transcriptase (Thermo Fisher) according to the manufacturer's instructions. To perform allele-expression experiments we used a modified version of a previously described method for quantifying methylation in bisulphite sequence data [82]. A two-stage PCR was used, the first round primers were designed to flank the variant of interest using Primer3 (http://bioinfo.ut.ee/primer3-0.4.0/primer3/) and adaptor sequences were added to the primers (Sigma), shown as lowercase letters (rs61839660_ASE_F tgtaaaacgacggccagt GCACACACCTATCCTAGCCT, rs61839660_ASE_R cag gaaacagctatgaccCCCACAGAATCACCCACTCT, product size 114 bp; rs12244380_ASE_F tgtaaaacgacggccagtTTCG TGGGAGTTGAGAGTGG, rs12244380_ASE_R caggaaac agctatgaccTTAAAAGAGTTCGCTGGGCC, product size 180 bp; rs12722495_ASE_F tgtaaaacgacggccagtGTGAGT TTCAATCCTAAGTGCGA, rs12722495_ASE_R cagga aacagctatgaccATTAAGCGGACTCTCTGGGG, product size $97 \mathrm{bp})$. The first-round PCR contains $10 \mu \mathrm{L}$ of Qiagen multiplex PCR mastermix, $0.5 \mu \mathrm{L}$ of $10 \mathrm{nmol}$ forward primer, $0.5 \mu \mathrm{L}$ of $10 \mathrm{nmol}$ reverse primer, $4 \mu \mathrm{L}$ of cDNA and made up to $20 \mu \mathrm{L}$ with ultra-pure water. The PCR cycling conditions were $95{ }^{\circ} \mathrm{C}$ for $15 \mathrm{~min}$ hot start, followed by 30 cycles of the following steps: $95{ }^{\circ} \mathrm{C}$ 
for $30 \mathrm{~s}, 60{ }^{\circ} \mathrm{C}$ for $90 \mathrm{~s}$ and $72{ }^{\circ} \mathrm{C}$ for $60 \mathrm{~s}$, finishing with a $72{ }^{\circ} \mathrm{C}$ for 10 -min cycle. The first-round PCR product was cleaned using AmpureXP beads (Beckman Coulter) according to manufacturer's instructions. To add Illumina sequence compatible ends to the individual first-round PCR amplicons, additional primers were designed to incorporate $\mathrm{P} 1$ and A sequences plus sample-specific index sequences in the A primer, through hybridisation to adapter sequence present on the first-round gene-specific primers. Index sequences are as published [82]. The second-round PCR contained $8 \mu \mathrm{L}$ of Qiagen multiplex PCR mastermix, $2.0 \mu \mathrm{L}$ of ultra-pure water, $0.35 \mu \mathrm{L}$ of each forward and reverse index primer and $5.3 \mu \mathrm{L}$ of Ampure XP-cleaned first-round PCR product. The PCR cycling conditions were $95{ }^{\circ} \mathrm{C}$ for $15 \mathrm{~min}$ hot start, followed by seven cycles of the following steps: $95{ }^{\circ} \mathrm{C}$ for $30 \mathrm{~s}, 56{ }^{\circ} \mathrm{C}$ for $90 \mathrm{~s}, 72{ }^{\circ} \mathrm{C}$ for $60 \mathrm{~s}$, finishing with $72{ }^{\circ} \mathrm{C}$ for a $10-\mathrm{min}$ cycle. All PCR products were pooled at equimolar concentrations based on quantification on the Shimadzu Multina. AmpureXP beads were used to remove unincorporated primers from the product pool. We used the Kapa Bioscience library quantification kit to accurately quantify the library according to the manufacturer's instructions before sequencing on an Illumina MiSeq v3 reagents $(2 \times 300$ bp reads).

\section{Statistical analysis of allele-specific expression data}

Sequence data were processed using the Methpup package (https://github.com/ollyburren/Methpup) to extract counts of each allele at rs12722495 and rs12244380 (Additional file 15: Table S10). Individuals were part of a larger cohort genotyped on the ImmunoChip and were phased using snphap (https://github.com/chr1swallace/snphap) to confirm which allele at each SNP was carried on the same chromosome as A2 $=$ rs12722495: $\mathrm{C}$ or A1 $=$ rs12722495: $\mathrm{T}$. Allelic imbalance was quantified as the ratio A2/A1 and was averaged across replicates within individuals using a geometric mean. Allelic ratios in cDNA and gDNA were compared using Wilcoxon rank sum tests. $p$ values are shown in Fig. 6b and Additional file 2: Figure S14. Full details are in https://github.com/chr1swallace/cd4-pchic/ blob/master/ASE/IL2RA-ASE.R.

\section{Additional files}

Additional file 1: Table S1. Gene modules inferred from WGCNA analysis of microarray time-course. Expression fold changes and associated false discovery rates (adjusted $p$ values) are from RNA-seq data at the 4-h timepoint. (GZ 278 kb)

Additional file 2: Figures S1-S14. Figure S1. Comparison of longer and shorter $\mathrm{CD} 4+\mathrm{T}$ cell activation timecourses. Figure S2. Summary distributions of interacting fragments. Figure S3. Validation of $\mathrm{PCHi}-\mathrm{C}$ by ChIA-PET. Figure S4. Chromatin state profiles of interacting fragments. Figure 55. Relationship of gene expression to PIR number and mRNA half-life. Figure S6. Definition and quantification of regulatory RNAs.
Figure S7. blockshifter calibration. Figure S8. MDN1 is prioritised for RA through ImmunoChip but not GWAS data. Figure S9. Gene prioritisation using COGS. Figure S10. Multiple genes on

chromosome 1q32.1 (IL10, IL19, IL20, IL24, FCAMR/PIGR) are prioritised for T1D, CRO and UC. Figure S11. Histograms show the distribution of summed PIR length by gene in non-activated CD4+ T cells (top panel) and TAD length in naive CD4+ T cells. Figure S12. IRF8 and EMC8/COX4/1 on chromosome 16 are prioritised for RA and SLE. Figure S13. AHR on chromosome 7 is prioritised for RA in activated CD4+ T cells. Figure S14. Allelic imbalance in mRNA expression in individuals heterozygous for group A SNPs is confirmed with reporter SNP rs12244380 (IL2RA 3' UTR). (PDF 4243 kb)

Additional file 3: Table S2. Results of differential expression analysis on RNA-seq data. Features are defined in the GTF file in Additional file 11 Table S8a. (GZ 835 kb)

Additional file 4: Table S3. Baited Hindlll fragments used for capture of Hi-C libraries, annotated with Ensembl annotated genes. (GZ $572 \mathrm{~kb}$ )

Additional file 5: Table S4. PCHi-C interactions called with the CHiCAGO pipeline. Annotation for baited fragments is given in Additional file 4: Table S3. PIRs are called other ends ('oe'). CHICAGO scores for activated (Total_CD4_Activated') and non-activated (Total_CD4_NonActivated') CD4+ T cells were considered called with confidence if above 5 . We also conducted differential analysis, and the read counts input into that are given by the columns P1.non - P3.act, with the results summarised by their log fold change (logFC) and FDR. Bait-PIR pairs are shown only if the CHiCAGO score is $\geq 5$ for at least one CD4+ T cell. (GZ $14529 \mathrm{~kb}$ )

Additional file 6: Table 55. Summary of GWAS data used. 'type' indicates whether the trait was quantitative (QUANT) or case/control (CC). For CC, 'cases' and 'controls' columns represent the number of individuals included in the study, while for QUANT, the number of individuals is given in the cases column. 'Category' indicates broader classes of traits. (XSLX $10 \mathrm{~kb}$ )

Additional file 7: Table S6a. Results of ImmunoChip fine-mapping by GUESSFM. (GZ $2833 \mathrm{~kb}$ )

Additional file 8: Table S6b. Results of GWAS summary statistic fine-mapping. (GZ $2833 \mathrm{~kb}$ )

Additional file 9: Table S7a. Autoimmune disease COGS gene prioritisation. Overall COGS gene scores (COGS_Overall_Gene_Score) for each gene and autoimmune disease are shown together with the prioritised category and score associated with that category (COGS Category Gene Score) (Fig. 3). The 'analysis' column describes whether the input data was GWAS or ImmunoChip (ICHIP) and whether summary statistic (SS) or GUESSFM (GF) fine-mapping was used. 'diff.expr' indicates whether the gene was not expressed (NA) or, if expressed, whether there was differential expression at the FDR $<0.01$ level (up, down or nsig). Similarly, 'diff.erna' indicates whether the Hindlll fragment containing the strongest SNP signal is differentially expressed with the same categories. Using data from ImmunoBase (https:// www.immunobase.org - accessed 06/06/2016), we annotate genes near (within $5 \mathrm{Mb}$ ) previously reported disease susceptibility regions, with contextual annotation 'Closest_Disease_Susceptibility_Region', 'Closest_ Min_P_Value_Susceptibility_SNP', 'Closest_Min_P_Value_Susceptibility_SNP_P_ Value', 'PIR_Overlaps_Disease_DSR' indicates that the PIR driving the prioritisation for a gene/disease overlaps an ImmunoBase known disease susceptibility region for that trait. Restricted to the subset of genes with scores $>0.5$ that are analysed in this paper. (GZ $37 \mathrm{~kb}$ )

Additional file 10: Table S7b. As above, complete results. (GZ 37 kb) Additional file 11: Table S8a. GTF file with definitions for all Ensembl 75 genomic features plus CD4-specific regulatory regions inferred from chromatin states. These regulatory regions have been named with identifiers containing a CD4R prefix, assigned a regulatory biotype and marked as pertaining to both genomic strands due to their bi-directional transcription potential. (GZ $39807 \mathrm{~kb}$ )

Additional file 12: Table S8b. Whole-genome segmentation of non-activated and activated CD4 T cells into 15 states obtained from a CHROMHMM analysis using ChIP-seq data for activated $C D 4^{+} T$ cells. (GZ $1551 \mathrm{~kb})$ 
Additional file 13: Table S8c. Whole-genome segmentation of non-activated and activated CD4 T cells into 15 states obtained from a CHROMHMM analysis using ChIP-seq data for non-activated CD4 ${ }^{+} \mathrm{T}$ cells. (GZ 1520 kb)

Additional file 14: Table S9. Genotypes for donors in the IL2RA ASE experiment across SNP groups A, C, D, E, F. (XLSX $12 \mathrm{~kb})$

Additional file 15: Table S10. Read counts for each allele at the IL2RA ASE experiment. The column Expt denotes sample id; time, the timepoint $(0,120,240 \mathrm{~min})$; stim, the condition (genomic DNA, time0 cDNA, stimulated or unstimulated cells cDNA). (GZ 4 kb)

\section{Acknowledgements}

We thank all study participants and family members.

We thank the Wellcome Trust for funding the AITD UK national collection; all doctors and nurses in Birmingham, Bournemouth, Cambridge, Cardiff, Exeter, Leeds, Newcastle and Sheffield for recruitment of patients; and J. Franklyn, S. Pearce (Newcastle) and P. Newby (Birmingham) for preparing and providing DNA samples on Graves' disease patients.

This research utilises resources provided by the Type 1 Diabetes Genetics Consortium, a collaborative clinical study sponsored by the National Institute of Diabetes and Digestive and Kidney Diseases (NIDDK), National Institute of Allergy and Infectious Diseases (NIAID), National Human Genome Research Institute (NHGRI), National Institute of Child Health and Human Development (NICHD), and JDRF and supported by U01 DK062418.

We gratefully acknowledge the participation of all Cambridge NIHR BioResource volunteers and thank the Cambridge BioResource staff for their help with volunteer recruitment. We thank the National Institute for Health Research Cambridge Biomedical Research Centre and NHS Blood and Transplant for funding. Further information can be found at www.cambridgebioresource.org.uk.

We thank the High-Throughput Genomics Group at the Wellcome Trust Centre for Human Genetics (funded by Wellcome Trust grant reference 090532/Z/09/Z) for the generation of the sequencing data.

We thank Stephen Eyre for his helpful comments on the manuscript and N. Soranzo and the HaemGen consortium for sharing blood trait GWAS summary statistics

We acknowledge the assistance and support of the National Institute for Health Research (NIHR) Cambridge Biomedical Research Centre; Helen Stevens, Meeta Maisuria-Armer, Pamela Clarke, Gillian Coleman, Sarah Dawson, Simon Duley, Jennifer Denesha and Trupti Mistry for sample processing; Judy Brown, Lynne Adshead, Amie Ashley, Anna Simpson and Niall Taylor for laboratory administration and procurement support; Vin Everett and Sundeep Nanuwa for logistical and web development. We thank investigators of published ImmunoChip studies for making available their raw genotyping data (David van Heel, celiac disease; Stephen Eyre, rheumatoid arthritis; Matthew Simmonds, Stephen Gough, Jayne Franklyn, and Oliver Brand, autoimmune thyroid disease).

\section{Funding}

This work was funded by the JDRF (9-2011-253), the Wellcome Trust (089989, 091157, 107881), the UK Medical Research Council (MR/L007150/1, MC_UP_1302/5), the UK Biotechnology and Biological Sciences Research Council (BB/J004480/1) and the National Institute for Health Research (NIHR) Cambridge Biomedical Research Centre. The research leading to these results has received funding from the European Union's 7th Framework Programme (FP7/2007-2013) under grant agreement no. 241447 (NAIMIT). The Cambridge Institute for Medical Research (CIMR) is in receipt of a Wellcome Trust Strategic Award (100140).

\section{Availability of data and materials}

The datasets generated and/or analysed during the current study are available in repositories or additional files as indicated below:

- PCHi-C data are available as raw sequencing reads from EGA (https://www.ebi.ac.uk/ega) accession number EGAS00001001911 or CHICAGO-called interactions (Additional file 5: Table S4) and are available for interactive exploration via https://www.chicp.org
- RNA-seq and ChIPseq data are available as raw sequencing reads from EGA (https://www.ebi.ac.uk/ega) accession number EGAS00001001961

- Microarray data are available at ArrayExpress, https://www.ebi.ac.uk/ arrayexpress, accession number E-MTAB-4832

- Processed datasets are available as Additional files

- Code used to analyse the data are available from https://github.com/ chr1swallace/cd4-pchic except where other URLs are indicated in 'Methods'. The version of scripts used in the production of this manuscript are archived under https://doi.org/10.5281/ zenodo.832849

\section{Authors' contributions}

Study conceived by: CW, JAT, LSW, PF and led by CW. Interpreted the data: CW, OSB, AJC, ARG, DR, LSW, JAT. Sequence data analysis: ARG. HiCUP analysis: SW. ASE experiments and analysis: DR. Microarray experiments and analysis: XCD, RCF, RC, CW. Statistical analysis: OSB, JC, NJC, CW, ARG. Laboratory experiments: AJC, BJ, DR, JJL, FB, SPR, KD. Wrote the paper: CW, OSB, AJC, ARG and contributed to writing: JAT, LSW, MS. Revised the paper: all authors. Genetic association data processing: CW, OSB, ES. Supervised capture Hi-C experiments: MS and PF. Supervised cell experiments: AJC, MF, WO, PF, JAT and LW. All authors read and approved the final manuscript.

\section{Ethics approval and consent to participate}

All samples and information were collected with written and signed informed consent. The study was approved by the local Peterborough and Fenland research ethics committee for the project entitled: 'An investigation into genes and mechanisms based on genotype-phenotype correlations in type 1 diabetes and related diseases using peripheral blood mononuclear cells from volunteers that are part of the Cambridge BioResource project' (05/Q0106/20). Experimental methods comply with the Helsinki Declaration.

\section{Consent for publication}

Not applicable

\section{Competing interests}

The authors declare that they have no competing interests.

\section{Author details}

'Department of Medicine, University of Cambridge, Addenbrooke's Hospital, Cambridge CB2 OSP, UK. ${ }^{2}$ JDRF/Wellcome Trust Diabetes and Inflammation Laboratory, Department of Medical Genetics, NIHR Cambridge Biomedical Research Centre, Cambridge Institute for Medical Research, University of Cambridge, Cambridge CB2 OXY, UK. ${ }^{3}$ Present address: JDRF/Wellcome Trust Diabetes and Inflammation Laboratory, Wellcome Trust Centre for Human Genetics, Nuffield Department of Medicine, NIHR Oxford Biomedical Research Centre, University of Oxford, Roosevelt Drive, Oxford OX3 7BN, UK. ${ }^{4}$ Nuclear Dynamics Programme, The Babraham Institute, Babraham Research Campus, Cambridge CB22 3AT, UK. ${ }^{5}$ Department of Haematology, University of Cambridge, Cambridge Biomedical Campus, Long Road, Cambridge CB2 OPT UK. ${ }^{6}$ National Health Service Blood and Transplant, Cambridge Biomedical Campus, Long Road, Cambridge CB2 OPT, UK. Bitish Heart Foundation Centre of Excellence, Division of Cardiovascular Medicine, Addenbrooke's Hospital, Hills Road, Cambridge CB2 0QQ, UK. ${ }^{8}$ Department of Human Genetics, Wellcome Trust Sanger Institute, Wellcome Trust Genome Campus, Hinxton, Cambridge CB10 1HH, UK. ${ }^{9}$ MRC Biostatistics Unit, University of Cambridge, Cambridge Institute of Public Health, Cambridge Biomedical Campus, Cambridge CB2 OSR, UK.

Received: 6 March 2017 Accepted: 21 July 2017

Published online: 04 September 2017

\section{References}

1. Maurano MT, Humbert R, Rynes E, Thurman RE, Haugen E, Wang $H$, et al. Systematic localization of common disease-associated variation in regulatory DNA. Science. 2012;337:1190-5. doi:10.1126/science.1222794.

2. Smemo S, Tena JJ, Kim K-H, Gamazon ER, Sakabe NJ, Gómez-Marín C, et al. Obesity-associated variants within FTO form long-range functional connections with IRX3. Nature. 2014;507:371-5. doi:10.1038/nature13138.

3. McGovern A, Schoenfelder S, Martin P, Massey J, Duffus K, Plant D, et al. Capture Hi-C identifies a novel causal gene, IL20RA, in the pan-autoimmune 
genetic susceptibility region 6q23. Genome Biol. 2016;17:212. doi:10.1186/ s13059-016-1078-x.

4. Xu Z, Zhang G, Duan Q, Chai S, Zhang B, Wu C, et al. HiView: an integrative genome browser to leverage $\mathrm{Hi}-\mathrm{C}$ results for the interpretation of GWAS variants. BMC Res Notes. 2016;9:159. doi:10.1186/s13104-016-1947-0.

5. Dryden NH, Broome LR, Dudbridge F, Johnson N, Orr N, Schoenfelder $S$, et al. Unbiased analysis of potential targets of breast cancer susceptibility loci by Capture Hi-C. Genome Res. 2014;24:1854-68. doi: 10.1101/gr.175034.114

6. Martin P, McGovern A, Orozco G, Duffus K, Yarwood A, Schoenfelder S, et al. Capture $\mathrm{Hi}-\mathrm{C}$ reveals novel candidate genes and complex long-range interactions with related autoimmune risk loci. Nat Commun. 2015;6:10069. doi:10.1038/ncomms10069.

7. Hnisz D, Abraham BJ, Lee TI, Lau A, Saint-André V, Sigova AA, et al. Super-enhancers in the control of cell identity and disease. Cell. 2013; 155:934-47. doi:10.1016/j.cell.2013.09.053.

8. Farh KK-H, Marson A, Zhu J, Kleinewietfeld M, Housley WJ, Beik S, et al. Genetic and epigenetic fine mapping of causal autoimmune disease variants. Nature. 2015;518:337-43. doi:10.1038/nature13835.

9. Coit P, Jeffries M, Altorok N, Dozmorov MG, Koelsch KA, Wren JD, et al. Genome-wide DNA methylation study suggests epigenetic accessibility and transcriptional poising of interferon-regulated genes in naïve CD4+ T cells from lupus patients. J Autoimmun. 2013;43:7884. doi:10.1016/j.jaut.2013.04.003.

10. Paul DS, Teschendorff AE, Dang MAN, Lowe R, Hawa MI, Ecker S, et al. Increased DNA methylation variability in type 1 diabetes across three immune effector cell types. Nat Commun. 2016;7:13555. doi:10.1038/ ncomms 13555 .

11. Benacerraf $B, M c D e v i t t ~ H O$. Histocompatibility-linked immune response genes. Science. 1972;175:273-9. http://www.ncbi.nlm.nih.gov/pubmed/4109878.

12. Gustafsson M, Gawel DR, Alfredsson L, Baranzini S, Björkander J, Blomgran R, et al. A validated gene regulatory network and GWAS identifies early regulators of T cell-associated diseases. Sci Transl Med. 2015;7:313ra178. doi: 10.1126/scitranslmed.aad2722.

13. Subramanian A, Tamayo P, Mootha VK, Mukherjee S, Ebert BL, Gillette MA, et al. Gene set enrichment analysis: a knowledge-based approach for interpreting genome-wide expression profiles. Proc Natl Acad Sci U S A. 2005;102:15545-50. doi:10.1073/pnas.0506580102.

14. Cairns J, Freire-Pritchett P, Wingett SW, Várnai C, Dimond A, Plagnol V, et al. CHiCAGO: robust detection of DNA looping interactions in Capture Hi-C data. Genome Biol. 2016;17:127. doi:10.1186/s13059-016-0992-2.

15. Chepelev I, Wei G, Wangsa D, Tang Q, Zhao K. Characterization of genome-wide enhancer-promoter interactions reveals co-expression of interacting genes and modes of higher order chromatin organization. Cell Res. 2012:22:490-503. doi:10.1038/cr.2012.15.

16. Vahedi G, Kanno Y, Furumoto Y, Jiang K, Parker SCJ, Erdos MR, et al. Superenhancers delineate disease-associated regulatory nodes in T cells. Nature. 2015;520:558-62. doi:10.1038/nature14154.

17. Javierre BM, Burren OS, Wilder SP, Kreuzhuber R, Hill SM, Sewitz S, et al. Lineage-specific genome architecture links enhancers and non-coding disease variants to target gene promoters. Cell. 2016;167:1369-84.e19. doi: 10.1016/j.cell.2016.09.037.

18. Raghavan A, Ogilvie RL, Reilly C, Abelson ML, Raghavan S, Vasdewani J, et al, Genome-wide analysis of mRNA decay in resting and activated primary human T lymphocytes. Nucleic Acids Res. 2002;30:5529-38. http://www.ncbi. nlm.nih.gov/pubmed/12490721.

19. Lam MTY, Li W, Rosenfeld MG, Glass CK. Enhancer RNAs and regulated transcriptional programs. Trends Biochem Sci. 2014;39:170-82. doi:10.1016/j. tibs.2014.02.007.

20. Ernst J, Kellis M. ChromHMM: automating chromatin-state discovery and characterization. Nat Methods. 2012;9:215-6. doi:10.1038/nmeth.1906.

21. Schmidl C, Hansmann L, Lassmann T, Balwierz PJ, Kawaji H, Itoh M, et al. The enhancer and promoter landscape of human regulatory and conventional T-cell subpopulations. Blood. 2014;123:e68-78. doi:10.1182/ blood-2013-02-486944.

22. Andersson R, Gebhard C, Miguel-Escalada I, Hoof I, Bornholdt J, Boyd M, et al. An atlas of active enhancers across human cell types and tissues. Nature. 2014:507:455-61. doi:10.1038/nature12787.

23. Li W, Notani D, Rosenfeld MG. Enhancers as non-coding RNA transcription units: recent insights and future perspectives. Nat Rev Genet. 2016;17:207-23. doi:10.1038/nrg.2016.4.
24. Levine M, Cattoglio C, Tjian R. Looping back to leap forward: transcription enters a new era. Cell. 2014;157:13-25. doi:10.1016/j.cell.2014.02.009.

25. Trynka G, Westra H-J, Slowikowski K, Hu X, Xu H, Stranger BE, et al. Disentangling the effects of colocalizing genomic annotations to functionally prioritize non-coding variants within complex-trait loci. Am J Hum Genet. 2015;97:139-52. doi:10.1016/.j.jhg.2015.05.016.

26. Wallace C, Cutler AJ, Pontikos N, Pekalski ML, Burren OS, Cooper JD, et al. Dissection of a complex disease susceptibility region using a Bayesian stochastic search approach to fine mapping. PLoS Genet. 2015;11, e1005272. doi:10.1371/journal.pgen.1005272.

27. Bowes J, Budu-Aggrey A, Huffmeier U, Uebe S, Steel K, Hebert HL, et al. Dense genotyping of immune-related susceptibility loci reveals new insights into the genetics of psoriatic arthritis. Nat Commun. 2015;6:6046. doi:10.1038/ncomms7046.

28. Trynka G, Hunt KA, Bockett NA, Romanos J, Mistry V, Szperl A, et al. Dense genotyping identifies and localizes multiple common and rare variant association signals in celiac disease. Nat Genet. 2011;43:1193-201. doi:10.1038/ng.998.

29. Smyth DJ, Plagnol V, Walker NM, Cooper JD, Downes K, Yang JHM, et al. Shared and distinct genetic variants in type 1 diabetes and celiac disease. N Engl J Med. 2008;359:2767-77. doi:10.1056/NEJMoa0807917.

30. Caballero-Franco C, Kissler S. The autoimmunity-associated gene RGS1 affects the frequency of T follicular helper cells. Genes Immun. 2016;17:22838. doi:10.1038/gene.2016.16.

31. Ferreira RC, Simons HZ, Thompson WS, Cutler AJ, Dopico XC, Smyth DJ, et al. IL-21 production by CD4+ effector T cells and frequency of circulating follicular helper $\mathrm{T}$ cells are increased in type 1 diabetes patients. Diabetologia. 2015;58:781-90. doi:10.1007/s00125-015-3509-8.

32. Nan L, Jacko AM, Tan J, Wang D, Zhao J, Kass DJ, et al. Ubiquitin carboxylterminal hydrolase-L5 promotes TGF $\beta$-1 signaling by de-ubiquitinating and stabilizing Smad2/Smad3 in pulmonary fibrosis. Sci Rep. 2016;6:33116. doi: $10.1038 /$ srep33116.

33. Wicks SJ, Haros K, Maillard M, Song L, Cohen RE, Dijke PT, et al. The deubiquitinating enzyme UCH37 interacts with Smads and regulates TGF-beta signalling. Oncogene. 2005;24:8080-4. doi:10.1038/sj.onc.1208944.

34. Hung T, Pratt GA, Sundararaman B, Townsend MJ, Chaivorapol C, Bhangale $T$, et al. The Ro60 autoantigen binds endogenous retroelements and regulates inflammatory gene expression. Science. 2015;350:455-9. doi:10. 1126/science.aac7442.

35. Onengut-Gumuscu S, Chen W-M, Burren O, Cooper NJ, Quinlan AR, Mychaleckyj JC, et al. Fine mapping of type 1 diabetes susceptibility loci and evidence for colocalization of causal variants with lymphoid gene enhancers. Nat Genet. 2015;47:381-6. doi:10.1038/ng.3245.

36. Moore KW, de Waal MR, Coffman RL, O'Garra A. Interleukin-10 and the interleukin-10 receptor. Annu Rev Immunol. 2001;19:683-765. http://www. annualreviews.org/doi/abs/10.1146/annurev.immunol.19.1.683.

37. Gaublomme JT, Yosef N, Lee Y, Gertner RS, Yang LV, Wu C, et al. Single-cell genomics unveils critical regulators of Th17 cell pathogenicity. Cell. 2015; 163:1400-12. doi:10.1016/j.cell.2015.11.009.

38. Hughes JR, Roberts N, McGowan S, Hay D, Giannoulatou E, Lynch M, et al. Analysis of hundreds of cis-regulatory landscapes at high resolution in a single, high-throughput experiment. Nat Genet. 2014;46:205-12. doi:10.1038/ng.2871.

39. Martin P, McGovern A, Massey J, Schoenfelder S, Duffus K, Yarwood A, et al. Identifying causal genes at the multiple sclerosis associated region 6q23 using Capture Hi-C. PLoS One. 2016;11, e0166923. doi:10. 1371/journal.pone.0166923.

40. Schmitt AD, Hu M, Jung I, Xu Z, Qiu Y, Tan CL, et al. A compendium of chromatin contact maps reveals spatially active regions in the human genome. Cell Rep. 2016;17:2042-59. doi:10.1016/j.celrep. 2016.10.061.

41. Meddens CA, Harakalova M, van den Dungen NAM, Foroughi Asl $H_{\text {, }}$ Hijma HJ, Cuppen EPJG, et al. Systematic analysis of chromatin interactions at disease associated loci links novel candidate genes to inflammatory bowel disease. Genome Biol. 2016;17:247. doi:10.1186/ s13059-016-1100-3.

42. Dendrou CA, Plagnol V, Fung E, Yang JHM, Downes K, Cooper JD, et al. Cell-specific protein phenotypes for the autoimmune locus IL2RA using a genotype-selectable human bioresource. Nat Genet. 2009;41:1011-5. doi:10.1038/ng.434.

43. Hambleton S, Salem S, Bustamante J, Bigley V, Boisson-Dupuis S, Azevedo J, et al. IRF8 mutations and human dendritic-cell immunodeficiency. N Engl J Med. 2011;365:127-38. doi:10.1056/NEJMoa1100066. 
44. Ouyang X, Zhang R, Yang J, Li Q, Qin L, Zhu C, et al. Transcription factor IRF8 directs a silencing programme for TH17 cell differentiation. Nat Commun. 2011;2:314. doi:10.1038/ncomms1311.

45. Patel DD, Kuchroo VK. Th17 cell pathway in human immunity: lessons from genetics and therapeutic interventions. Immunity. 2015;43:1040-51. doi:10. 1016/j.immuni.2015.12.003.

46. Nguyen NT, Nakahama T, Nguyen $\mathrm{CH}$, Tran TT, Le VS, Chu HH, et al. Aryl hydrocarbon receptor antagonism and its role in rheumatoid arthritis. J Exp Pharmacol. 2015;7:29-35. doi:10.2147/JEP.S63549.

47. Liao W, Lin J-X, Leonard WJ. Interleukin-2 at the crossroads of effector responses, tolerance, and immunotherapy. Immunity. 2013;38:13-25. doi:10. 1016/j.immuni.2013.01.004.

48. International Multiple Sclerosis Genetics Consortium, Wellcome Trust Case Control Consortium 2, Sawcer S, Hellenthal G, Pirinen M, Spencer CCA, et al. Genetic risk and a primary role for cell-mediated immune mechanisms in multiple sclerosis. Nature. 2011;476:214-9. doi:10.1038/nature10251.

49. Okada Y, Wu D, Trynka G, Raj T, Terao C, Ikari K, et al. Genetics of rheumatoid arthritis contributes to biology and drug discovery. Nature. 2014;506:376-81. doi:10.1038/nature12873.

50. Jostins L, Ripke S, Weersma RK, Duerr RH, McGovern DP, Hui KY, et al. Hostmicrobe interactions have shaped the genetic architecture of inflammatory bowel disease. Nature. 2012;491:119-24. doi:10.1038/nature1 1582.

51. Garg G, Tyler JR, Yang JHM, Cutler AJ, Downes K, Pekalski M, et al. Type 1 diabetes-associated IL2RA variation lowers IL-2 signaling and contributes to diminished CD4 + CD25+ regulatory T cell function. J Immunol. 2012;188: 4644-53. doi:10.4049/jimmunol.1100272.

52. Ballesteros-Tato A. Beyond regulatory T cells: the potential role for IL-2 to deplete T-follicular helper cells and treat autoimmune diseases. Immunotherapy. 2014;6:1207-20. doi:10.2217/imt.14.83.

53. Guo H, Fortune MD, Burren OS, Schofield E, Todd JA, Wallace C. Integration of disease association and eQTL data using a Bayesian colocalisation approach highlights six candidate causal genes in immune-mediated diseases. Hum Mol Genet. 2015;24:3305-13. doi:10.1093/hmg/ddv077.

54. Chen L, Ge B, Casale FP, Vasquez L, Kwan T, Garrido-Martín D, et al. Genetic drivers of epigenetic and transcriptional variation in human immune cells. Cell. 2016;167:1398-414.e24. doi:10.1016/j.cell.2016.10.026.

55. Zhernakova DV, Deelen $P$, Vermaat $M$, van Iterson $M$, van Galen $M$, Arindrarto $W$, et al. Identification of context-dependent expression quantitative trait loci in whole blood. Nat Genet. 2017;49:139-45. doi:10.1038/ng.3737.

56. Duhen T, Duhen R, Lanzavecchia A, Sallusto F, Campbell DJ. Functionally distinct subsets of human FOXP3+ Treg cells that phenotypically mirror effector Th cells. Blood. 2012;119:4430-40. doi:10. 1182/blood-2011-11-392324.

57. Ye CJ, Feng T, Kwon H-K, Raj T, Wilson MT, Asinovski N, et al. Intersection of population variation and autoimmunity genetics in human $\mathrm{T}$ cell activation. Science. 2014;345:1254665. doi:10.1126/science.1254665.

58. Bonder MJ, Luijk R, Zhernakova DV, Moed M, Deelen $\mathrm{P}$, Vermaat $\mathrm{M}$, et al. Disease variants alter transcription factor levels and methylation of their binding sites. Nat Genet. 2017;49:131-8. doi:10.1038/ng.3721.

59. Hua J, Davis SP, Hill JA, Yamagata T. Diverse gene expression in human regulatory T cell subsets uncovers connection between regulatory T cell genes and suppressive function. J Immunol. 2015;195:3642-53. doi:10.4049/ jimmunol.1500349,

60. Pekalski ML, Ferreira RC, Coulson RMR, Cutler AJ, Guo H, Smyth DJ, et al. Postthymic expansion in human CD4 naive T cells defined by expression of functional high-affinity IL-2 receptors. J Immunol. 2013;190:2554-66. doi:10. 4049/jimmunol.1202914.

61. Wright FA, Sullivan PF, Brooks Al, Zou F, Sun W, Xia K, et al. Heritability and genomics of gene expression in peripheral blood. Nat Genet. 2014;46:430-7. doi:10.1038/ng.2951.

62. Brown CD, Mangravite LM, Engelhardt BE. Integrative modeling of eQTLs and Cis-regulatory elements suggests mechanisms underlying cell type specificity of eQTLs. PLoS Genet. 2013;9, e1003649. doi:10.1371/journal.pgen.1003649.

63. Kumasaka N, Knights AJ, Gaffney DJ. Fine-mapping cellular QTLs with RASQUAL and ATAC-seq. Nat Genet. 2016;48:206-13. doi:10.1038/ng.3467.

64. Mifsud B, Tavares-Cadete F, Young AN, Sugar R, Schoenfelder S, Ferreira L, et al. Mapping long-range promoter contacts in human cells with highresolution capture Hi-C. Nat Genet. 2015:47:598-606. doi:10.1038/ng.3286.

65. Wingett $S$, Ewels $P$, Furlan-Magaril M, Nagano T, Schoenfelder S, Fraser $P$, et al. HiCUP: pipeline for mapping and processing Hi-C data. F1000Res. 2015:4:1310. doi:10.12688/f1000research.7334.1.
66. Ritchie ME, Phipson B, Wu D, Hu Y, Law CW, Shi W, et al. limma powers differential expression analyses for RNA-sequencing and microarray studies. Nucleic Acids Res. 2015;43:e47. doi:10.1093/nar/gkv007.

67. Huber W, von Heydebreck A, Sültmann H, Poustka A, Vingron M. Variance stabilization applied to microarray data calibration and to the quantification of differential expression. Bioinformatics. 2002;18 Suppl 1:S96-104. doi:10. 1093/bioinformatics/18.suppl_1.S96.

68. Langfelder P, Horvath S. WGCNA: an R package for weighted correlation network analysis. BMC Bioinf. 2008;9:559. doi:10.1186/1471-2105-9-559.

69. Li H, Durbin R. Fast and accurate long-read alignment with BurrowsWheeler transform. Bioinformatics. 2010;26:589-95. doi:10.1093/ bioinformatics/btp698.

70. Li H, Handsaker B, Wysoker A, Fennell T, Ruan J, Homer N, et al. The Sequence Alignment/Map format and SAMtools. Bioinformatics. 2009;25: 2078-9. doi:10.1093/bioinformatics/btp352.

71. Martin M. Cutadapt removes adapter sequences from high-throughput sequencing reads. EMB Net J. 2011:17:10-2. doi:10.14806/ej.17.1.200.

72. Dobin A, Davis CA, Schlesinger F, Drenkow J, Zaleski C, Jha S, et al. STAR: ultrafast universal RNA-seq aligner. Bioinformatics. 2013;29:15-21. doi:10. 1093/bioinformatics/bts635.

73. Anders S, Pyl PT, Huber W. HTSeq-a Python framework to work with high-throughput sequencing data. Bioinformatics. 2015;31:166-9. doi:10. 1093/bioinformatics/btu638.

74. Sanyal A, Lajoie BR, Jain G, Dekker J. The long-range interaction landscape of gene promoters. Nature. 2012;489:109-13. doi:10.1038/nature11279.

75. Giambartolomei C, Vukcevic D, Schadt EE, Franke L, Hingorani AD, Wallace C, et al. Bayesian test for colocalisation between pairs of genetic association studies using summary statistics. PLoS Genet. 2014;10, e1004383. doi:10. 1371/journal.pgen.1004383.

76. Wakefield J. Bayes factors for genome-wide association studies: comparison with P-values. Genet Epidemiol. 2009;33:79-86. http://onlinelibrary.wiley. com/doi/10.1002/gepi.20359/full.

77. The Wellcome Trust Case Control Consortium, Maller JB, McVean G, Byrnes J, Vukcevic D, Palin K, et al. Bayesian refinement of association signals for 14 loci in 3 common diseases. Nat Genet. 2012;44:1294-301. doi:10.1038/ng.2435.

78. Liu JZ, McRae AF, Nyholt DR, Medland SE, Wray NR, Brown KM, et al. A versatile gene-based test for genome-wide association studies. Am J Hum Genet. 2010;87:139-45. doi:10.1016/j.ajhg.2010.06.009.

79. Chapman JM, Cooper JD, Todd JA, Clayton DG. Detecting disease associations due to linkage disequilibrium using haplotype tags: a class of tests and the determinants of statistical power. Hum Hered. 2003;56:1831. doi:10.1159/000073729.

80. Stahl EA, Raychaudhuri S, Remmers EF, Xie G, Eyre S, Thomson BP, et al. Genome-wide association study meta-analysis identifies seven new rheumatoid arthritis risk loci. Nat Genet. 2010;42:508-14. doi:10.1038/ng.582.

81. Marchini J, Howie B, Myers S, McVean G, Donnelly P. A new multipoint method for genome-wide association studies by imputation of genotypes. Nat Genet. 2007:39:906-13. doi:10.1038/ng2088.

82. Rainbow DB, Yang X, Burren O, Pekalski ML, Smyth DJ, Klarqvist MDR, et al. Epigenetic analysis of regulatory $T$ cells using multiplex bisulfite sequencing. Eur J Immunol. 2015;45:3200-3. doi:10.1002/eji.201545646.

\section{Submit your next manuscript to BioMed Central and we will help you at every step:}

- We accept pre-submission inquiries

- Our selector tool helps you to find the most relevant journal

- We provide round the clock customer support

- Convenient online submission

- Thorough peer review

- Inclusion in PubMed and all major indexing services

- Maximum visibility for your research

Submit your manuscript at www.biomedcentral.com/submit 